\title{
A contact domain method for large deformation frictional contact problems. Part 2: Numerical aspects
}

\author{
S. Hartmann ${ }^{\text {a }}$, J. Oliver ${ }^{\mathrm{a}, *}$, R. Weyler ${ }^{\mathrm{b}}$, J.C. Cante ${ }^{\mathrm{b}}$, J.A. Hernández ${ }^{\mathrm{a}}$ \\ a E.T.S. d'Enginyers de Camins, Canals i Ports, Technical University of Catalonia (UPC), Campus Nord UPC, Mòdul C-1, c/Jordi Girona 1-3, 08034 Barcelona, Spain \\ ${ }^{\mathrm{b}}$ E.T.S. d'Enginyería Industrial i Aeronáutica de Terrassa, Technical University of Catalonia (UPC), Campus Nord UPC, Mòdul C-1, c/Jordi Girona 1-3, 08034 Barcelona, Spain
}

\section{A R T I C L E I N F O}

\section{Article history:}

Received 7 August 2008

Received in revised form 6 March 2009

Accepted 10 March 2009

Available online 27 March 2009

\section{Keywords:}

Contact domain

Interior penalty method

Stabilized Lagrange multiplier method

Frictional contact

Self contact

Dynamic contact

\begin{abstract}
A B S T R A C T
This second part of the work describes the numerical aspects of the developed contact domain method for large deformation frictional contact problems. The theoretical basis of this contact method is detailed in the first part of this work. Starting from this, the present contribution focuses on describing important algorithmic details that go along with the finite element implementation for two-dimensional problems. Important aspects are the construction of the contact domain mesh, via a constraint Delaunay triangulation, the linearization of the discretized contact contributions and some important technical aspects about the extrapolation procedure used for the predictive active set strategy. Finally a set of numerical examples is presented to demonstrate the performance of the developed contact strategy. Demanding static and dynamic contact problems in the context of large deformations, including frictional effects as well as self contact, show the wide applicability and the robustness of the proposed method.
\end{abstract}

(C) 2009 Elsevier B.V. All rights reserved.

\section{Introduction}

In the first part of this work [14] the theoretical aspects of the socalled contact domain method have been presented. The method uses a so-called contact domain to discretize the contact interface. This contact domain can be interpreted as a fictive intermediate region that connects the potential contact surfaces and has the same dimension as the deformable contacting bodies. The utilized contact domain is discretized with a non-overlapping set of patches that leads to a pairing of the finite element nodes in the contact boundaries. Based on this discretization scheme, the geometric normal and tangential contact constraints are formulated in terms of dimensionless, strain-like measures. Another important feature of the presented contact strategy is the introduction of a computational contact strategy that utilizes a stabilized Lagrange multiplier formulation for the enforcement of the contact constraints. This allows the condensation of the additional unknowns to end up with a purely displacement driven problem. For the determination of the active subsets of the contact domain, where the contact/friction restrictions have to be imposed, a predictive active set strategy is utilized.

Since this approach brings new and very specific ingredients with respect to more classical contact methods [11,17], the corresponding numerical and algorithmic treatment needs detailed attention. In this sense, and in order to provide the interested reader with suitable information to ensure the reproducibility of the

\footnotetext{
* Corresponding author.

E-mail address: xavier.oliver@upc.edu (J. Oliver)
}

method, this second part of the work concentrates on these numerical and algorithmic aspects, provides detailed information about its finite element implementation and supplies a representative set of examples displaying the performance of the proposed methodology in a number of different contact scenarios.

The remaining of this paper is organized as follows: Section 2 shortly describes the problem to be analyzed, summarizes some basic notations and restates the modified variational formulation utilized within the contact domain method derived in [14]. The construction of the contact domain mesh, which can in turn be interpreted as a contact searching strategy, is illustrated in Section 3. In Section 4 the spatial discretization of the contact virtual work and the contact constraint variational equations is given, followed by the necessary linearization of these contributions. Furthermore a matrix notation for the different elemental contact residuals and stiffness contributions is derived. Important technical aspects of the predictive active set strategy, introduced in [14] are described in Section 5. The performance of the proposed algorithm is analyzed in Section 6, by means of various numerical examples, including static and dynamic, frictionless and frictional, large deformation (self) contact problems. Finally, Section 7 provides some concluding remarks.

\section{Problem description}

In this section a frictional large deformation contact problem will be very briefly recalled to introduce some notations subsequently used in this paper. The contact domain, appropriate gap definitions and the modified variational problem of the frictional 
contact problem are summarized for the use of linear triangular patches for the approximation of the introduced contact domain. For a detailed derivation of these expressions, the reader is referred to the first part of this work [14].

\subsection{Basic notations}

Dealing with contact problems, one might need to face scenarios of numerous deformable bodies coming into contact with each other (multiple contact), as well as the possibility, that parts of the boundary of one specific body might come into contact with another part of the boundary of the same body (self contact). Without lacking generality, the subsequent description will be done on basis of one contact pair. A two-dimensional, large deformation contact problem of two deformable bodies $\Omega^{(\alpha)}, \alpha=1,2$, eventually coming into contact within a specific time step $\left[t_{n}, t_{n+1}\right]$ is shown in Fig. 1 , where

$\mathbf{u}^{(\alpha)}=\mathbf{x}_{n+1}^{(\alpha)}-\mathbf{x}_{n}^{(\alpha)} \quad \forall \mathbf{x}_{n}^{(\alpha)} \in \Omega_{n}^{(\alpha)}$

defines the incremental displacement field of the two contacting bodies.
The boundaries $\partial \Omega^{(\alpha)}$ of $\Omega^{(\alpha)}$ are divided into $\Gamma_{u}^{(\alpha)}$, where displacements are prescribed, $\Gamma_{\sigma}^{(\alpha)}$ where tractions are prescribed and a part $\Gamma_{D}^{(\alpha)}$ where the bodies might be in contact at the end of the time interval. It is assumed that the following conditions are satisfied:

$\Gamma_{u}^{(\alpha)} \cup \Gamma_{\sigma}^{(\alpha)} \cup \Gamma_{D}^{(\alpha)}=\partial \Omega^{(\alpha)}$ and

$\Gamma_{\sigma}^{(\alpha)} \cap \Gamma_{u}^{(\alpha)}=\Gamma_{\sigma}^{(\alpha)} \cap \Gamma_{D}^{(\alpha)}=\Gamma_{u}^{(\alpha)} \cap \Gamma_{D}^{(\alpha)}=\emptyset$.

Additionally a so-called contact domain $D_{n}$ is defined, joining the possible contact boundaries $\Gamma_{D}^{(\alpha)}$. This contact domain has the same dimension as the contacting bodies and will be approximated by a set of non-overlapping, linear triangular patches (see Fig. 1). Within this contact domain, yet another incremental displacement field $\mathbf{u}^{(D)}$ is defined, which describes the motion of any point within this domain. It is linearly interpolated from the corresponding incremental displacements at the contacting boundaries (see Part 1 [14], Section 2).

\subsection{Geometrical gap}

The normal and tangential gaps are defined for every point within the contact domain as the projection of the final gap vector $\mathbf{g}\left(\mathbf{x}_{n}\right)$ onto its current normal and tangent direction (see Fig. 2):

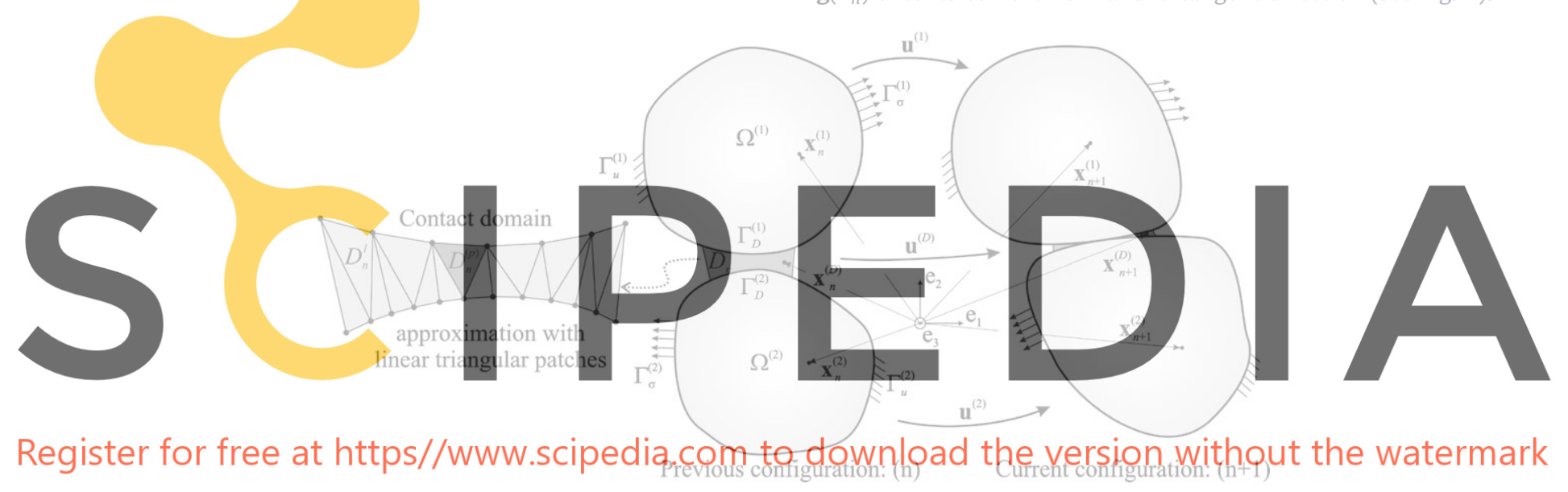

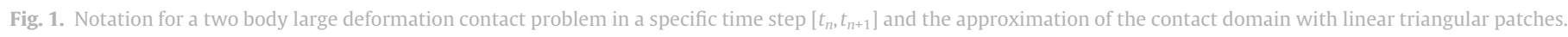

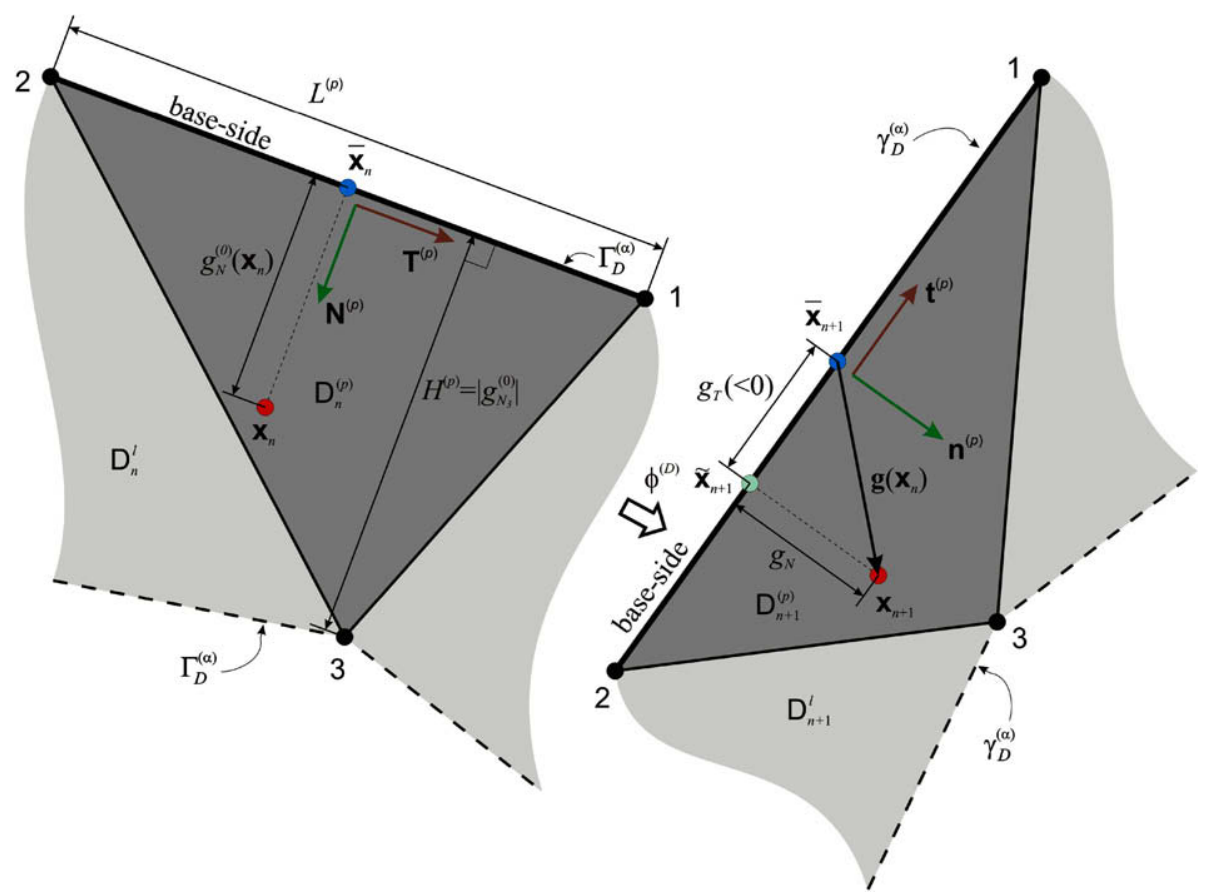

Fig. 2. Linear triangle contact patch in previous and current configuration. 


$$
\begin{aligned}
& g_{N}\left(\mathbf{x}_{n}\right)=\mathbf{n}^{(p)} \cdot \mathbf{g}\left(\mathbf{x}_{n}\right)=g_{N}^{(0)}\left(\mathbf{x}_{n}\right) \mathbf{n}^{(p)} \cdot \boldsymbol{f}^{(p)} \cdot \mathbf{N}^{(p)}, \\
& g_{T}\left(\mathbf{x}_{n}\right)=\mathbf{t}^{(p)} \cdot \mathbf{g}\left(\mathbf{x}_{n}\right)=g_{N}^{(0)}\left(\mathbf{x}_{n}\right) \mathbf{t}^{(p)} \cdot \boldsymbol{f}^{(p)} \cdot \mathbf{N}^{(p)} .
\end{aligned}
$$

Herein $\mathbf{n}^{(p)}$ and $\mathbf{t}^{(p)}$ are the current patch-wise constant normal and tangential vectors, $\mathbf{N}^{(p)}$ is the reference (material) normal vector and $\boldsymbol{f}^{(p)}$ is the incremental gradient of deformation tensor, defined with

$\boldsymbol{f}^{(p)}=\mathbf{1}+G R A D\left(\mathbf{u}^{(p)}\right)$,

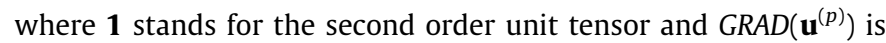
the material gradient of the incremental displacement field within a contact patch. Furthermore $g_{N}^{(0)}\left(\mathbf{x}_{n}\right)$ is the initial normal gap of a point $\mathbf{x}_{n} \in D_{n}^{(p)}$ (see Fig. 2).

\subsection{Gap intensities}

A distinguishing feature of the proposed method, is the usage of dimensionless, strain-like measures to formulate the geometrical contact constraints. Therefore the so-called normal and tangential gap intensities

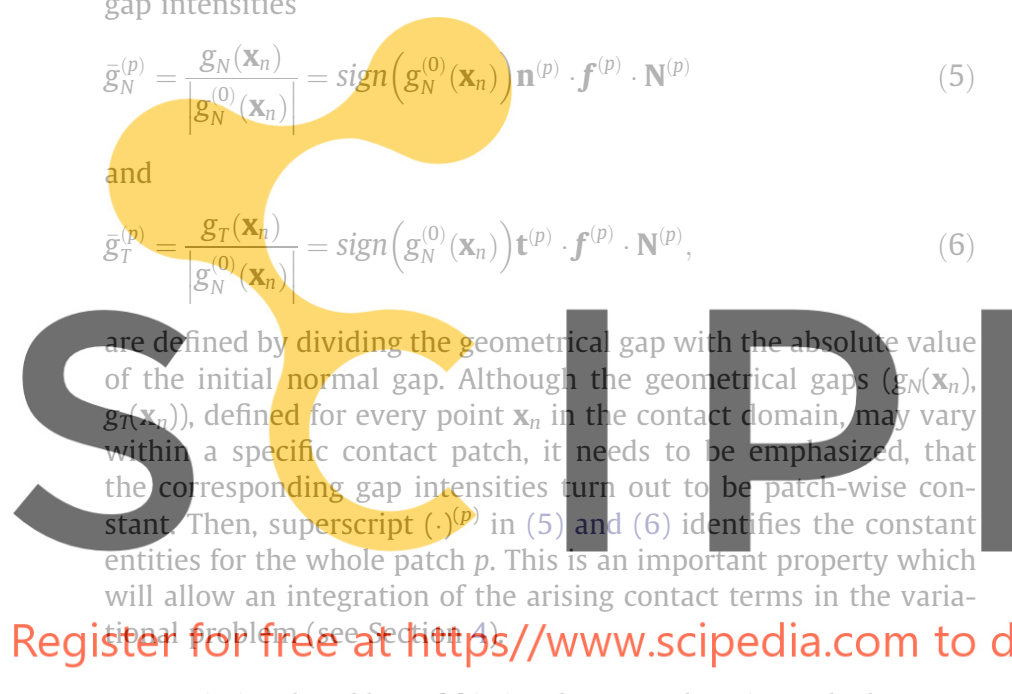

\subsection{Variational problem of frictional contact domain method}

Applying a finite element discretization scheme to solve contact problems, an appropriate weak form of the underlying boundary value problem is needed. The contact domain method presented in [14] enforces the contact constraints using a generalization of the stabilized Lagrange multiplier method used in [8]. This allows for the condensation of the introduced Lagrange multipliers $\left(\lambda_{N}, \lambda_{T}\right)$, which represent the normal and tangential contact tractions at the contacting boundaries.

It is assumed that the active contact and friction domains, fulfilling the appropriate constraints at the end of the considered time step are known in advance by means of an active set strategy (see Part 1 [14], Section 5), which allows to translate the initial inequality constrained problem into an equality constrained one (see Part 1 [14], Section 3). The active normal contact domain $D_{n}^{(N)} \subset D_{n}^{l}$ is then further subdivided into a part $D_{n}^{(T)}$, where stick conditions have to be applied, and into the remaining part $D_{n}^{(N)} \backslash D_{n}^{(T)}$, where slip conditions, fulfilling an appropriate friction law, have to be enforced. Using appropriate spaces for the incremental displacements, $\mathbf{u}=\left[\mathbf{u}^{(\alpha)}, \mathbf{u}^{(D)}\right]$, and their variations, $\delta \mathbf{u}=\left[\delta \mathbf{u}^{(\alpha)}, \delta \mathbf{u}^{(D)}\right]$ (virtual displacements), as well as for the introduced Lagrange multipliers, $\lambda=\left[\lambda_{N}, \lambda_{T}\right]$, and their variations, $\delta \lambda=\left[\delta \lambda_{N}, \delta \lambda_{T}\right]$, the utilized variational equations can be summarized. The virtual work principle (see Part 1 [14], Section 4) reads

$\delta \Pi_{\text {mech }}(\mathbf{u}, \boldsymbol{\lambda}, \delta \mathbf{u}):=\delta \Pi_{\text {int }, \text { ext }}\left(\mathbf{u}^{(\alpha)}, \delta \mathbf{u}^{(\alpha)}\right)+\delta \Pi_{\text {cont }}\left(\mathbf{u}^{(D)}, \delta \mathbf{u}^{(D)}, \boldsymbol{\lambda}\right)=0$, where $\delta \Pi_{\text {mech }}$ is the total virtual mechanical work and $\delta \Pi_{\text {int,ext }}$ denotes the sum of the virtual work arising from the internal and external forces of the contacting bodies. Various variational energy principles can be used to derive an expression for the virtual work done by the internal and external forces of the respective body. As the present work concentrates on the description of the contact phenomena, this will not be detailed any further. The main focus of this work will be on the second contribution in Eq. (7), namely the contact virtual work. Utilizing the variations of the normal and tangential gap intensities, the contact virtual work expression can be written as

$$
\delta \Pi_{\text {cont }}\left(\mathbf{u}^{(D)}, \delta \mathbf{u}^{(D)}, \lambda\right)=\underbrace{\int_{D_{n}^{(N)}} \lambda_{N} \delta \bar{g}_{N} d D}_{\text {normal contact }}+\underbrace{\int_{D_{n}^{(T)}} \lambda_{T} \delta \bar{g}_{T} d D}_{\text {stick }}+\underbrace{\int_{D_{n}^{(N)} \backslash D_{n}^{(T)}} \mathscr{T} \delta \bar{g}_{T} d D}_{\text {slip }} .
$$

Herein, $\mathscr{T}=-\mu \operatorname{sign}\left(\bar{g}_{T}\right)\left|\lambda_{N}\right|$ represents the classical coulomb friction law, with $\mu$ being the coefficient of friction. As can be seen in Eq. (8), the contact virtual work expression consists of three different portions. An integral over the active normal contact domain $D_{n}^{(N)}$, the active stick domain $D_{n}^{(T)}$ and the slip domain $D_{n}^{(N)} \backslash D_{n}^{(T)}$, respectively. For the enforcement of the contact constraints, two additional variational equations (see Part 1 [14], Section 4)

\section{$\delta \Pi_{\lambda_{N}}\left(\mathbf{u}, \lambda_{N}, \delta \lambda_{N}\right)=\int_{D_{n}^{(N)}} \delta \lambda_{N} \bar{g}_{N} d D+\int_{\partial D_{n}^{(N)} \cap \Gamma_{D}^{(\alpha)}} \delta \lambda_{N} \tau\left(t_{N}-\lambda_{N}\right) d \Gamma=0$}

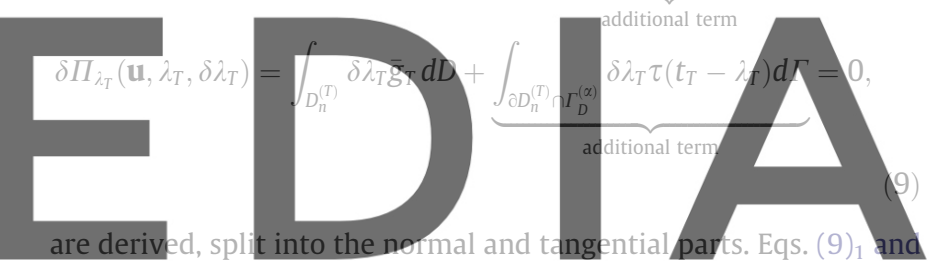

$(9)_{2}$ are the (stabilized) variational constraint equations to enforce the normal and frictional (stick) contact conditions within the

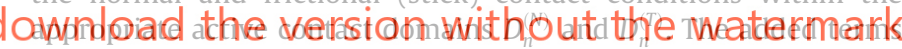
can be interpreted as stabilization terms, which allow the condensation of the introduced Lagrange multipliers, where $\tau$ is a user defined stabilization parameter, which will be discussed later. Furthermore, $t_{N}$ and $t_{T}$ are the projections of the traction vector at the boundaries of the contacting bodies onto the current normal and tangential directions, given with

$t_{N}=\mathbf{n} \cdot \mathbf{P} \cdot \mathbf{N}$ and $t_{T}=\mathbf{t} \cdot \mathbf{P} \cdot \mathbf{N}$,

where $\mathbf{P}$ is the first Piola-Kirchhoff stress tensor with respect to the previous configuration $\left(\Omega_{n}^{(\alpha)}\right)$. It is worth noting, that the normal and tangential tractions defined in Eq. (10) live in the boundary of the body $\Gamma_{D}^{(\alpha)}$ and not in the contact domain $D_{n}^{l}$.

\section{Contact pairing algorithm}

Finding the appropriate contact pairs is generally a difficult task, which has lead to various proposals for effective contact searching algorithms (e.g. [1,13,18,19]). Yang and Laursen [19] extend their contact searching algorithm for finite sliding mortar formulations [18] to the case of self contact. In order to achieve a comparable computational cost in their self contact searching algorithm, they need to add some special criterions to their basic algorithm. In this work, a general contact pairing strategy is presented, which can naturally be applied to any contact scenario, including self contact. It is based on a so-called constraint Delaunay triangulation, producing at the same time the necessary contact domain mesh between potential contact pairs. The technical aspects of this procedure are detailed in the following. 


\subsection{Construction of the contact domain mesh}

In Fig. 3 the basic steps of the construction of the contact domain mesh are shown. Fig. 3a displays two discretized bodies, eventually being in contact. The contact domain mesh to be constructed should have the property to connect the boundary nodes of the discretized bodies. Therefore all the interior nodes of the discretized bodies are removed. A key step in the generation process is the shrinkage of the outer boundary, taking into account the outward normals at the boundary nodes, which are computed as the weighted average of the normals of the adjacent boundary lines. That means, that every finite element node on the contact boundary is being offset in the negative direction of its outward normal by a user defined distance (shrinkage parameter), depending upon the element size. The removal of the interior nodes, as well as the shrinkage or offset of the boundary nodes is displayed in Fig. 3b. This shrinkage of the approximated boundaries of the contacting bodies has the effect, that the potential contacting surfaces of the solids are separated from each other in such a way, that an automatic mesh generation algorithm can generate a triangulation (domain mesh). Subsequently only the position of the modified boundary nodes and their specific outward normals are needed for the remaining steps. This information is then given to an automatic triangulation algorithm, which connects the boundary nodes. Another parameter (the alpha shape parameter [10]) is supplied to the mesher, such that only boundary nodes, that are sufficiently close to each other are connected. The result of this

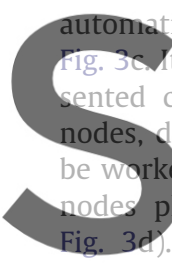
triangulation is the contac is worth noting, that for the i nted contact strategy, only the conn be worked with the original spatial posit Fig 3d. That means that the important step of repositioning the boundary nodes (shrinkage) is only a technical necessity to allow the meshing algorithm to build up the connectivity and

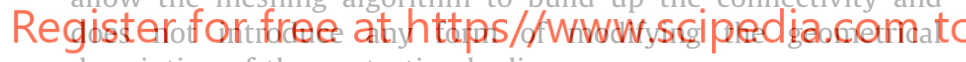
description of the contacting bodies.

(a)
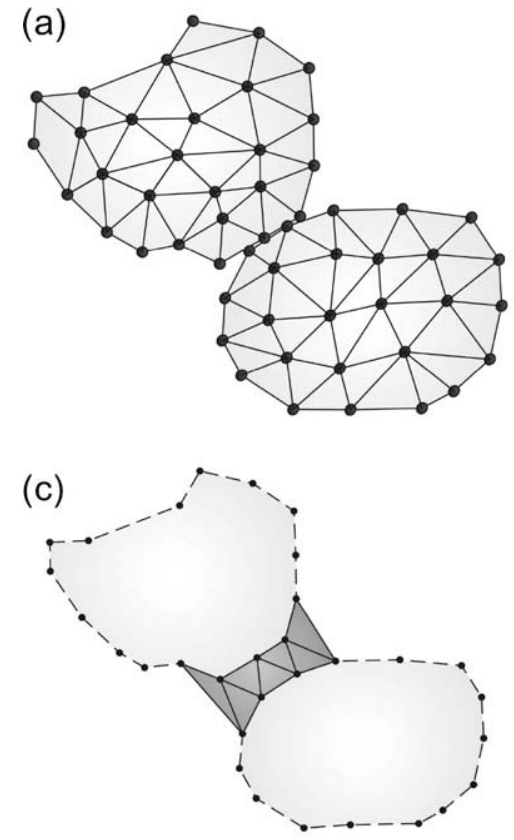

\section{Finite element approximation}

To solve the variational problem in Eq. (7), the deformable bodies $\Omega^{(\alpha)}$ as well as the contact domain $D_{n}$ are discretized using finite elements and contact patches. The discretization of the contacting bodies is done using standard, linear CST (constant strain triangle) elements. This allows an easy evaluation of the discrete variational constraint equations, as the stress field within such an element is constant (see Part 1 [14], Section 5).

\subsection{Discretization of the contact domain}

\subsubsection{Contact domain discretization}

As already introduced in the previous sections, the subdivision of the contact domain will be done on basis of linear, triangular elements (patches). A typical contact domain element is shown in Fig. 2 in the previous and current configuration. It is obvious, that every of these elements has one node (vertex) placed on the boundary of one contacting body and two on the boundary of the other. To ease the further documentation, the local numbering of the nodes within one contact element will be used as shown in Fig. 2. Therefore node 3 is always the individual node and the nodes 1 and 2 are placed on a shared edge with an adjacent finite element of a contacting body. Then the displacement field in the contact domain is approximated with

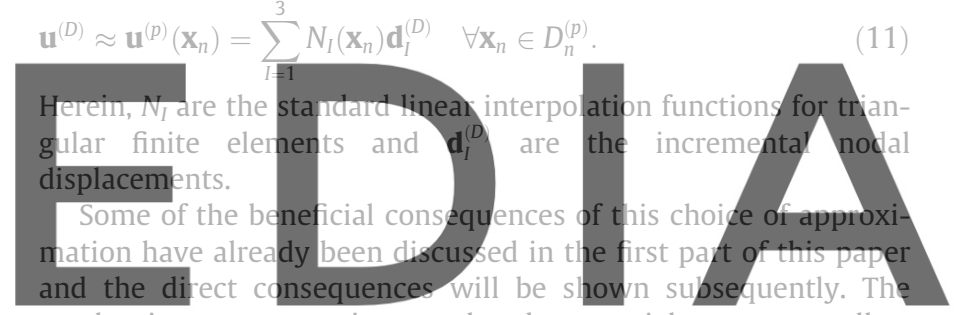
patch-wise constant unit normal and tangential vector as well as the length of the common edge (base-side) $L^{(p)}$ and the absolute

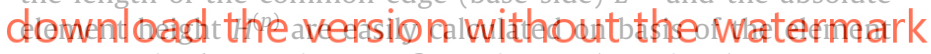
geometry in the previous configuration $\Omega_{n}$ (see Fig. 2):

(b)

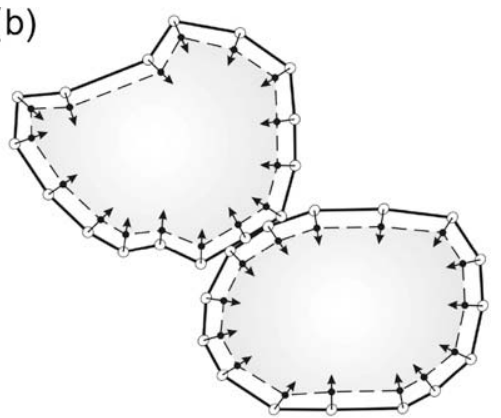

(d)

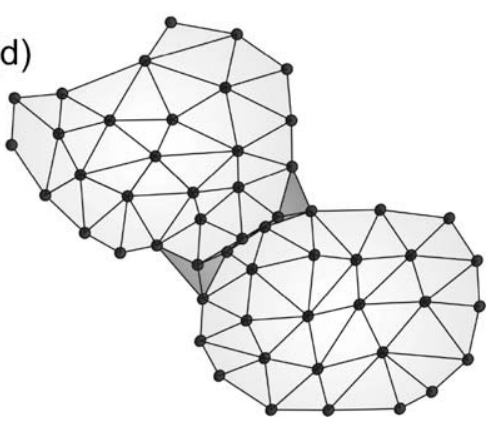

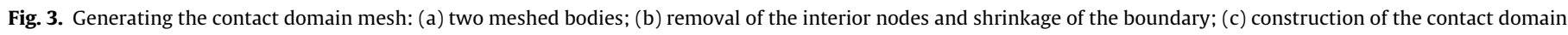
mesh; (d) retrieve of original boundary and mesh. 
$\mathbf{T}^{(p)}=\frac{1}{L^{(p)}}\left(\mathbf{x}_{1}-\mathbf{x}_{2}\right) \quad$ with $L^{(p)}=\left\|\mathbf{x}_{1}-\mathbf{x}_{2}\right\|$,

then using the definition

$\mathbf{N}^{(p)}=\mathbf{T}^{(p)} \times \hat{\mathbf{e}}_{3}$,

where $\hat{\mathbf{e}}_{3}$ stands for the out-of-plane unit vector leads to the unit normal vector

$\mathbf{N}^{(p)}=\left(\begin{array}{c}T_{2}^{(p)} \\ -T_{1}^{(p)}\end{array}\right)$

and finally the absolute element height is given with

$H^{(p)}=\left|\left(\mathbf{x}_{3}-\mathbf{x}_{1}\right) \cdot \mathbf{N}^{(p)}\right|$.

\subsubsection{Discretization of the Lagrange multipliers}

Using the modified variational equations (7) and (9), suitable approximations for the introduced normal and tangential Lagrange multipliers have to be chosen. Therefore piece-wise constant interpolation functions $\psi^{(p)}$ are used:
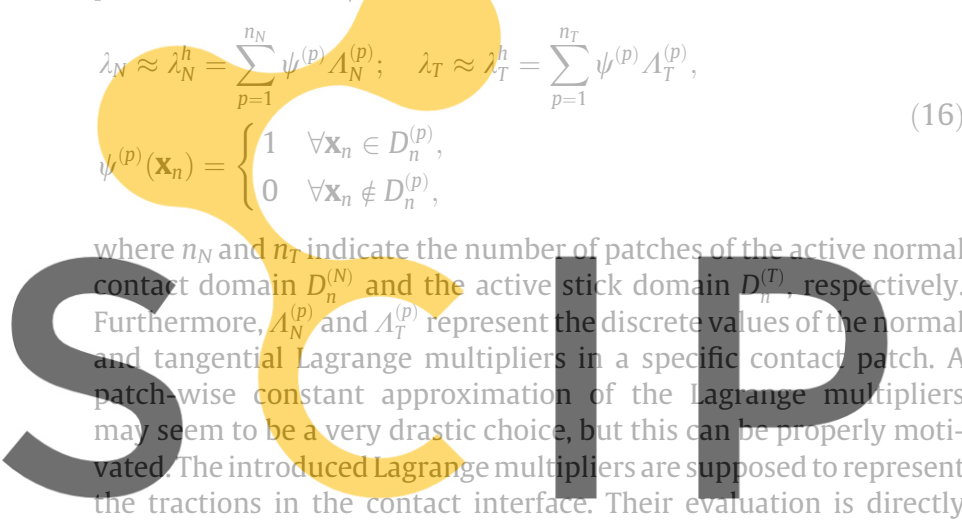
linked to the surface tractions $\left(t_{N}, t_{T}\right)$ in the contacting bodies through

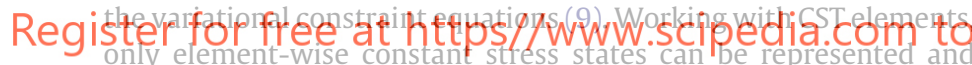
therefore, the surface tractions $\left(t_{N}, t_{T}\right)$ are going to be constant along the base-side of a contact patch, as the associated normal and tangential vectors are patch-wise constant. Furthermore, the employed gap intensities $\left(\bar{g}_{N}, \bar{g}_{T}\right)$, appearing in Eq. (9) are constant within a contact patch. Therefore the introduced Lagrange multipliers can only represent constant contact tractions and consequently a patch-wise constant approximation is a suitable choice.

As the subsequent explanations are based on one contact domain element (patch), the super-/subscripts $(\cdot)_{n}^{(p)}$ will be omitted in the following.

\subsection{Local constraint enforcement}

Due to the element-wise approximation of the Lagrange multipliers, the enforcement of the contact constraints in Eq. (9) can be decoupled and thus enforced separately for every single contact domain element. In fact, every single contact domain element can be interpreted as an individual contact domain itself, such that the definition of the normal and tangential vectors does not need to be conforming across the elements. Furthermore, one contact domain element, which is identified as being active for normal contact, has either to fulfill a stick or a slip condition. Assuming that a specific contact domain element needs to enforce a stick condition, the following two constraints have to be fulfilled:

$$
\begin{aligned}
& \delta \Pi_{\lambda_{N}}^{h}\left(\mathbf{d}, \Lambda_{N}, \delta \Lambda_{N}\right)=\int_{D} \delta \Lambda_{N} \bar{g}_{N} d D+\int_{\Gamma} \delta \Lambda_{N} \tau\left(t_{N}-\Lambda_{N}\right) d \Gamma=0, \\
& \delta \Pi_{\lambda_{T}}^{h}\left(\mathbf{d}, \Lambda_{T}, \delta \Lambda_{T}\right)=\int_{D} \delta \Lambda_{T} \bar{g}_{T} d D+\int_{\Gamma} \delta \Lambda_{T} \tau\left(t_{T}-\Lambda_{T}\right) d \Gamma=0 .
\end{aligned}
$$

Herein $\Gamma$ stands for the base-side of the contact element and $t_{N}$ and $t_{T}$ are the normal and tangential tractions in the finite element of the contacting body sharing the common edge (the base-side) with the contact domain element (see Eq. (10)). As the spatial discretization of the contacting bodies is done with CST elements, the stress field in the solids is constant within one finite element and therefore the tractions are constant along the common edge. Furthermore the normal and tangential gap intensities $\bar{g}_{N}$ and $\bar{g}_{T}$ are constant within one contact domain element (see Eqs. (5) and (6)). Finally, due to the element-wise constant approximation of the normal and tangential Lagrange multipliers in Eq. (16) and a patch-wise constant stabilization parameter $\tau$, the integration of the discrete variational constraint equations $(17)_{1+2}$ can be done analytically. Using

$\int_{D} d D=\frac{1}{2} L H \quad$ and $\quad \int_{\Gamma} d \Gamma=L$

where $L$ is the length of the base-side and $H$ is the absolute height of the reference triangular patch (see Fig. 2), which in turn displays the absolute value of the initial normal gap $\left(g_{N}^{(0)}\right)_{3}=g_{N}^{(0)}\left(\mathbf{x}_{3}\right)$ of the vertex node 3

$H=\left|g_{N}^{(0)}\left(\mathbf{x}_{3}\right)\right|=\left|\left(g_{N}^{(0)}\right)_{3}\right|$

Eq. $(17)_{1+2}$ can be solved for the elemental normal and tangential Lagrange multipliers

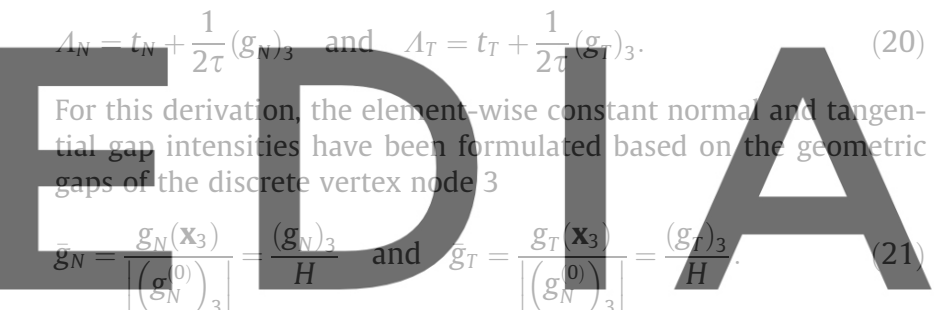

In here, $\left(g_{N}\right)_{3}=g_{N}\left(\mathbf{X}_{3}\right)$ and $\left(g_{T}\right)_{3}=g_{T}\left(\mathbf{X}_{3}\right)$ denote the geometrical nordammlaamgthen velssiqhe witthode the wdater:mark

$$
\begin{aligned}
\left(g_{N}\right)_{3} & =\left(g_{N}^{(0)}\right)_{3} \mathbf{n} \cdot(\mathbf{N}+G R A D(\mathbf{u}) \cdot \mathbf{N}) \\
& =\left(g_{N}^{(0)}\right)_{3} \mathbf{n} \cdot \mathbf{N}+\left(g_{N}^{(0)}\right)_{3} \mathbf{n} \cdot G R A D(\mathbf{u}) \cdot \mathbf{N}, \\
\left(g_{T}\right)_{3} & =\left(g_{N}^{(0)}\right)_{3} \mathbf{t} \cdot(\mathbf{N}+G R A D(\mathbf{u}) \cdot \mathbf{N}) \\
& =\left(g_{N}^{(0)}\right)_{3} \mathbf{t} \cdot \mathbf{N}+\left(g_{N}^{(0)}\right)_{3} \mathbf{t} \cdot G R A D(\mathbf{u}) \cdot \mathbf{N} .
\end{aligned}
$$

\subsection{Exact evaluation of the discrete constraint equations}

Taking a closer look at the normal and tangential gap definitions in Eq. (22) might give rise to some concerns. What happens if the initial normal gap $\left(g_{N}^{(0)}\right)$ of the vertex node 3 , actually measuring the distance between contacting boundaries, tends to zero? The first part of the dot product will tend to zero, but at the same time, the gradient of the displacement field in normal direction will tend to infinity. In the following it will be shown that the second part of the gap definitions in Eq. (22) can be exactly computed, independently of the value of the initial normal gap $\left(g_{N}^{(0)}\right)_{3}$. Using

$\operatorname{GRAD}(\mathbf{u}) \cdot \mathbf{N}=\frac{\partial \mathbf{u}}{\partial N}$

and the linear discretization of the displacement field within a contact domain element in Eq. (11), the interesting part of Eq. (22) can be written as:

$\left(g_{N}^{(0)}\right)_{3} G R A D(\mathbf{u}) \cdot \mathbf{N}=\left(g_{N}^{(0)}\right)_{3} \sum_{I=1}^{3} \frac{\partial N_{I}}{\partial N} \mathbf{d}_{I}$. 
In Fig. 4 a geometrical interpretation of the first part in Eq. (24) is shown. Multiplying the shape functions with the initial normal gap $\left(g_{N}^{(0)}\right)$ allows for a direct evaluation of this term, which is totally independent of $\left(g_{N}^{(0)}\right)_{3}$ :

$$
\left(g_{N}^{(0)}\right)_{3} \frac{\partial N_{1}}{\partial N}=-\frac{A}{L} ; \quad\left(g_{N}^{(0)}\right)_{3} \frac{\partial N_{2}}{\partial N}=-\frac{B}{L} ; \quad\left(g_{N}^{(0)}\right)_{3} \frac{\partial N_{3}}{\partial N}=1 .
$$

Therefore the evaluation of this term can be cut down to a pure geometrical problem. It is only necessary to get the ratios of how the normal projection of node 3 intersects the base-line, which is defined by the nodes 1 and 2 in the previous configuration. This can be uniquely evaluated no matter of how distorted the contact domain element is, even if the normal projection of node 3 onto the base-line lies outside of the edge 1-2 (see Fig. 5). Thus the normal and tangential gaps (see Eq. (22)) can be evaluated with:

$$
\begin{aligned}
& \left(g_{N}\right)_{3}=\left(g_{N}^{(0)}\right)_{3} \mathbf{n} \cdot \mathbf{N}+\mathbf{n} \cdot \sum_{I=1}^{3}\left(\left(g_{N}^{(0)}\right)_{3} \frac{\partial N_{I}}{\partial N}\right) \mathbf{d}_{I}, \\
& \left(g_{T}\right)_{3}=\left(g_{N}^{(0)}\right)_{3} \mathbf{t} \cdot \mathbf{N}+\mathbf{t} \cdot \sum_{I=1}^{3}\left(\left(g_{N}^{(0)}\right)_{3} \frac{\partial N_{I}}{\partial N}\right) \mathbf{d}_{I}
\end{aligned}
$$

\subsection{Contact contributions}

After the determination of the discrete Lagrange multipliers, by enforcing the contact constraints, the resulting contact contributions can be computed. Therefore the contact virtual work expression in Eq. (8) is discretized using the introduced approximations. To shorten the expressions, the contact contributions will be split into a normal contact, a stick and a slip part, respectively. In the following they will be specified for one contact patch.

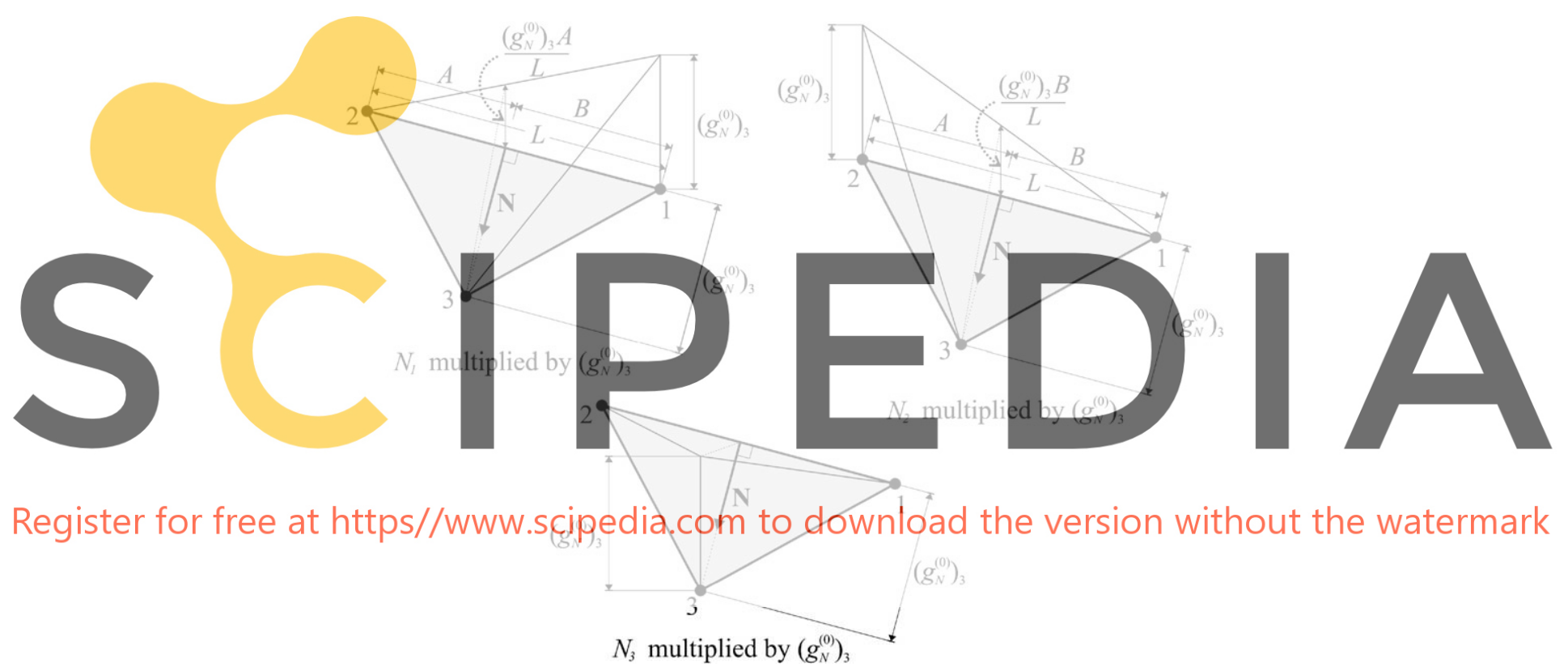

Fig. 4. Normal direction derivatives of the shape functions (regular element) in the previous configuration.

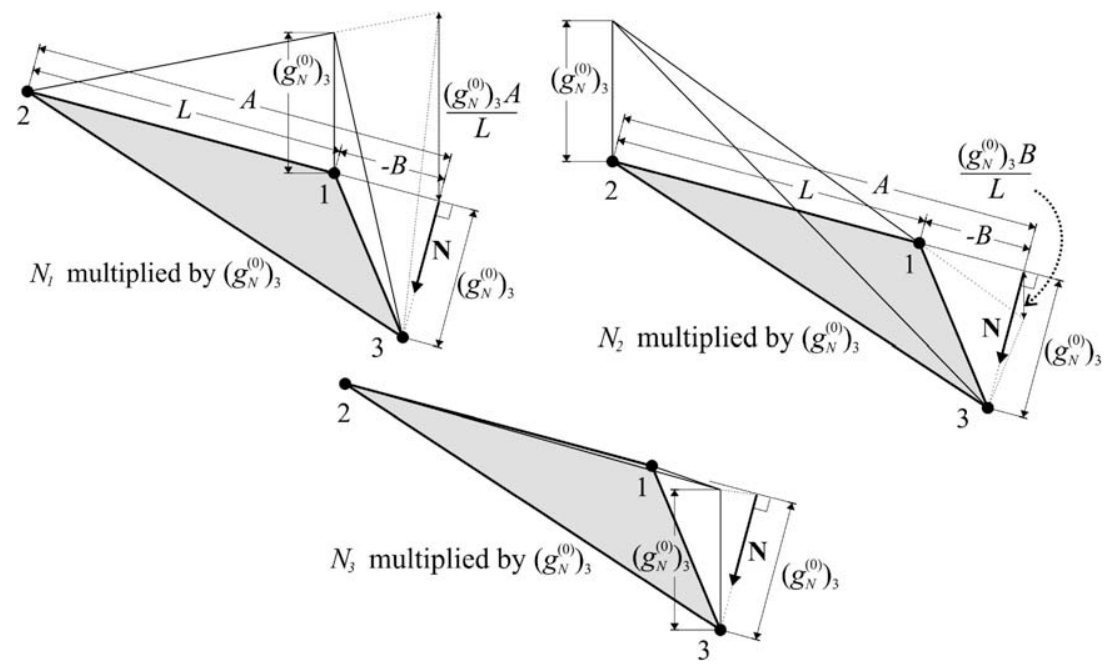

Fig. 5. Normal direction derivatives of the shape functions (distorted element) in the previous configuration. 
4.4.1. Normal contact

The normal contribution of the contact virtual work expression (8) is given with

$\delta \Pi_{\text {cont }}^{(N)}=\int_{D} \lambda_{N} \delta \bar{g}_{N} d D$.

Its evaluation necessitates the variation of the normal gap intensity, which has been derived in the Appendix of the first part of this paper [14]. It is given with

$\delta \overline{\mathrm{g}}_{N}=\overline{\mathrm{g}}_{N} \mathbf{n} \cdot \operatorname{grad}(\delta \mathbf{u}) \cdot \mathbf{n}$,

which again is constant within one contact domain element. Therefore Eq. (27) can again be evaluated analytically using Eq. (18) ${ }_{1}$. Introducing the spatial approximation and using Eq. (21) finally leads to

$\delta \Pi_{\text {cont }}^{(N)}=\frac{L}{2} \Lambda_{N} \mathbf{n} \cdot \sum_{I-1}^{3}\left(\left(g_{N}\right)_{3} \frac{\partial N_{I}}{\partial \eta}\right) \cdot \delta \mathbf{d}_{I}$.

Similarly to what has been shown for the expressions in Eq. (25) holds for the terms appearing in brackets in (29). They can be evaluated on basis of the current geometrical properties of the contact domain element with

$\left(g_{N}\right)_{3} \frac{\partial N_{1}}{\partial n}=-\frac{a}{l} ; \quad\left(g_{N}\right)_{3} \frac{\partial N_{2}}{\partial n}=-\frac{b}{l} ; \quad\left(g_{N}\right)_{3} \frac{\partial N_{3}}{\partial n}=1$

where $l$ is the length of the base-side of a contact domain element

and the fractions $a$ and $b$ have the same meaning as $A$ and $B$ (see Figs. 4 and 5), but now evaluated in its

4.4.2. Tangential contact - stick par

ff a contact domain element is active in stick, the following con-

ibution of the virtual work expression (8) needs to be considered:

$\delta \Pi_{\text {cont }}^{(T)}=\int_{D} \lambda_{T} \delta \bar{g}_{T} d D$

(31)

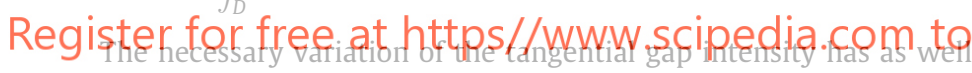
been derived in the Appendix of the first part of this paper [14]. It reads

$\delta \bar{g}_{T}=\bar{g}_{N}(\mathbf{n} \cdot \operatorname{grad}(\delta \mathbf{u}) \cdot \mathbf{t}+\mathbf{t} \cdot \operatorname{grad}(\delta \mathbf{u}) \cdot \mathbf{n})+\bar{g}_{T} \mathbf{t} \cdot \operatorname{grad}(\delta \mathbf{u}) \cdot \mathbf{t}$

and is again constant within a contact domain element. Inserting the finite element approximations and using Eqs. (18) 1 and (21) yields the stick contribution to the contact virtual work for one contact patch:

$$
\begin{aligned}
\delta \Pi_{\text {cont }}^{(T)}= & \frac{L}{2} \Lambda_{T}\left[\left(g_{N}\right)_{3} \mathbf{n} \cdot \sum_{I=1}^{3}\left(\frac{\partial N_{I}}{\partial t}\right) \cdot \delta \mathbf{d}_{I}+\mathbf{t} \cdot \sum_{I=1}^{3}\left(\left(g_{N}\right)_{3} \frac{\partial N_{I}}{\partial n}\right) \cdot \delta \mathbf{d}_{I}\right. \\
& \left.+\left(g_{T}\right)_{3} \mathbf{t} \cdot \sum_{I=1}^{3}\left(\frac{\partial N_{I}}{\partial t}\right) \cdot \delta \mathbf{d}_{I}\right] .
\end{aligned}
$$

Herein, the tangential directional derivatives of the shape functions are given with (see Fig. 6)

$\frac{\partial N_{1}}{\partial t}=\frac{1}{l} ; \quad \frac{\partial N_{2}}{\partial t}=-\frac{1}{l} ; \quad \frac{\partial N_{3}}{\partial t}=0$.

\subsubsection{Tangential contact - slip part}

The slip contribution of the virtual work expression (8) reads

$\delta \Pi_{\text {cont }}^{(N \backslash T)}=\int_{D} \mathscr{T} \delta \bar{g}_{T} d D$.

Applying the finite element approximations and using Eqs. (32), $(18)_{1}$ and (21) leads to

$$
\begin{aligned}
\delta \Pi_{\text {cont }}^{(N \backslash T)}= & \frac{L}{2} \mathscr{T}\left[\left(g_{N}\right)_{3} \mathbf{n} \cdot \sum_{I=1}^{3}\left(\frac{\partial N_{I}}{\partial t}\right) \cdot \delta \mathbf{d}_{I}+\mathbf{t} \cdot \sum_{I=1}^{3}\left(\left(g_{N}\right)_{3} \frac{\partial N_{I}}{\partial n}\right) \cdot \delta \mathbf{d}_{I}\right. \\
& \left.+\left(g_{T}\right)_{3} \mathbf{t} \cdot \sum_{I=1}^{3}\left(\frac{\partial N_{I}}{\partial t}\right) \cdot \delta \mathbf{d}_{I}\right]
\end{aligned}
$$

with

$\mathscr{T}=-\mu \operatorname{sign}\left(\bar{g}_{T}\right)\left|\Lambda_{N}\right|$.

\subsection{Effective structural equation}

Starting from the variational form (7) of the entire problem the semi-discrete equation of motion is derived introducing the spatial discretization of the contacting bodies as well as the approximations within the contact domain. This leads to a compact notation of the semi-discrete initial value problem (see part 1 [14], Section 4):

$\mathrm{G}_{\text {int,ext }}(\mathbf{d})+\mathbf{G}_{\text {cont }}(\mathbf{d}, \mathbf{\Lambda})=0$

Herein

$$
\mathbf{G}_{\text {int }, e x t}(\mathbf{d})=\mathbf{M} \ddot{\mathbf{d}}+\mathbf{F}_{\text {int }}(\mathbf{d})-\mathbf{F}_{\text {ext }}
$$

is the discretized counterpart of the virtual work of the two contacting bodies, where $\mathbf{M}$ is the mass matrix, $\mathbf{F}_{\text {int }}(\mathbf{d})$ is the vector of deformation dependent internal forces, $\mathbf{F}_{\text {ext }}$ is the vector of external forces and $\mathbf{d}$ and $\ddot{d}$ represent the discrete nodal displacements

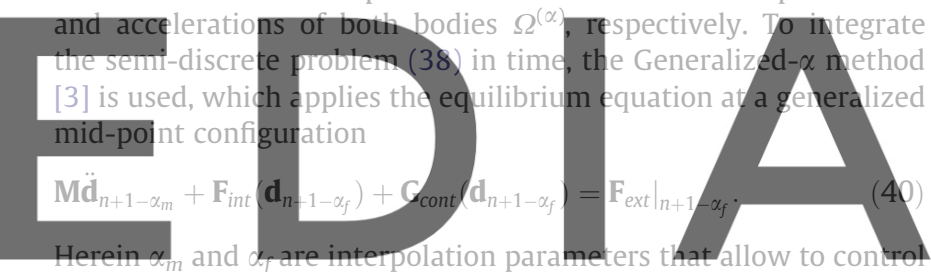

the numerical dissipation. Inserting the classical Newmark approximatipns [12linto (40), leads to a folly discretized non-linear equaThe necessary linearization yields the effective incremental structural equation to be solved within every iteration $k$ :

$\mathbf{K}_{T}^{\text {eff }} \Delta \mathbf{d}=\mathbf{G}^{\text {eff }}$,

where

$$
\begin{aligned}
\mathbf{K}_{T}^{e f f}= & \frac{1-\alpha_{m}}{\beta \Delta t^{2}} \mathbf{M}+\left(1-\alpha_{f}\right) \frac{\partial \mathbf{F}_{i n t}\left(\mathbf{d}_{n+1}^{(k)}\right)}{\partial \mathbf{d}_{n+1}^{(k)}} \\
& +\left(1-\alpha_{f}\right) \underbrace{\frac{\partial \mathbf{G}_{\text {cont }}\left(\mathbf{d}_{n+1}^{(k)}\right)}{\partial \mathbf{d}_{n+1}^{k}}}_{\mathbf{K}_{\text {cont }}}
\end{aligned}
$$

is the effective tangential stiffness matrix and

$$
\begin{aligned}
\mathbf{G}^{\text {eff }}= & \left.\mathbf{F}_{\text {ext }}\right|_{n+1-\alpha_{f}}-\frac{1-\alpha_{m}}{\beta \Delta t^{2}} \mathbf{M} \mathbf{d}_{n+1}^{(k)}+\mathbf{h}\left(\mathbf{d}_{n}, \dot{\mathbf{d}}_{n}, \ddot{\mathbf{d}}_{n}\right) \\
& -\mathbf{F}_{\text {int }}\left(\mathbf{d}_{n+1-\alpha_{f}}\right)-\mathbf{G}_{\text {cont }}\left(\mathbf{d}_{n+1-\alpha_{f}}\right)
\end{aligned}
$$

is the effective residual vector. Herein $\mathbf{h}\left(\mathbf{d}_{n}, \dot{\mathbf{d}}_{n}, \ddot{\mathbf{d}}_{n}\right)$ is a history term, that only depends on given state variables at time $t_{n}$ :

$\mathbf{h}\left(\mathbf{d}_{n}, \dot{\mathbf{d}}_{n}, \ddot{\mathbf{d}}_{n}\right)=\mathbf{M}\left[\frac{1-\alpha_{m}}{\beta \Delta t^{2}} \mathbf{d}_{n}+\frac{1-\alpha_{m}}{\beta \Delta t} \dot{\mathbf{d}}_{n}+\frac{1-\alpha_{m}-2 \beta}{2 \beta} \ddot{\mathbf{d}}_{n}\right]$.

\subsection{Linearization of contact contributions}

The construction of the effective tangential stiffness matrix in Eq. (42) necessitates, amongst others, the linearization of the con- 

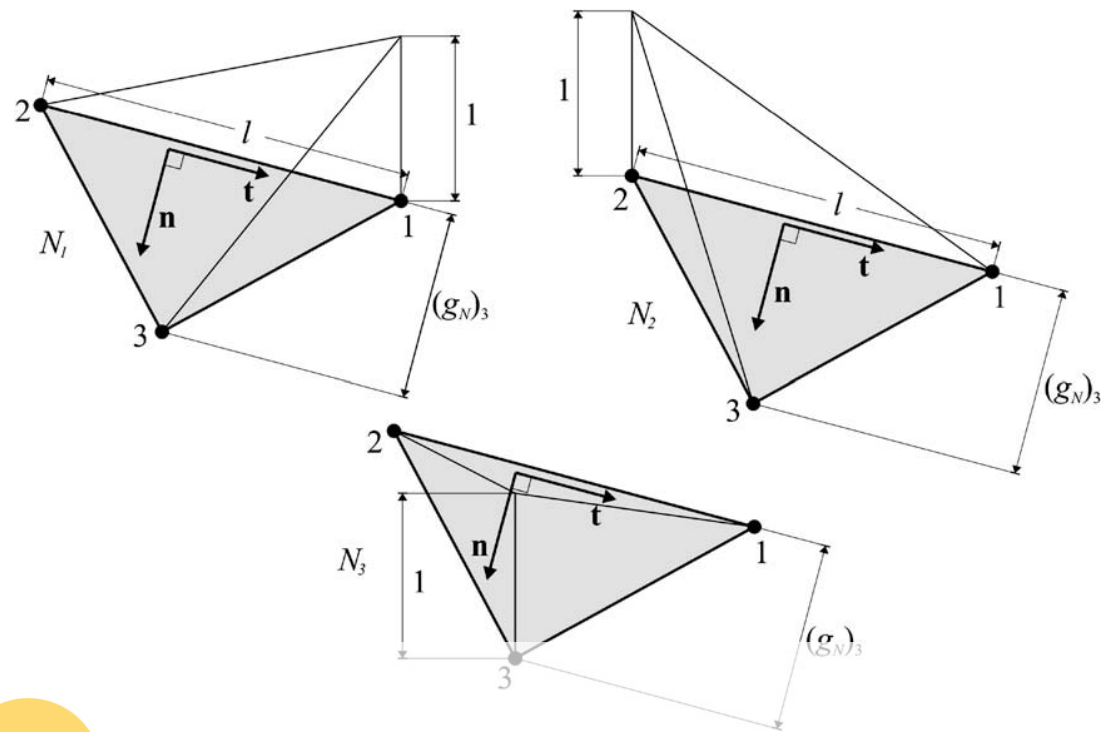

tact virtual work expression (8). In analogy to Section 4.4, this linearization will be specified separately for the different contact contributions for one individual contact patch.

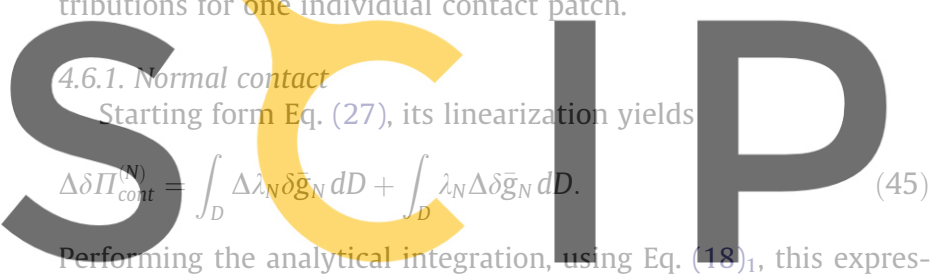

sion can be written for one contact domain element with

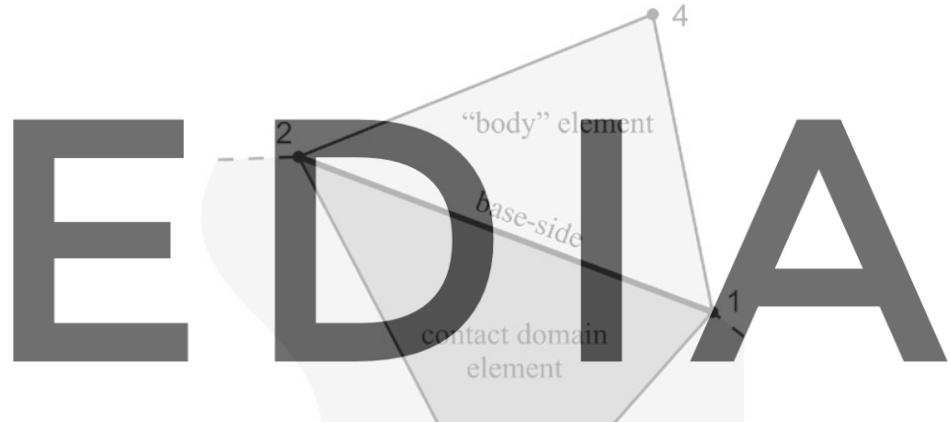

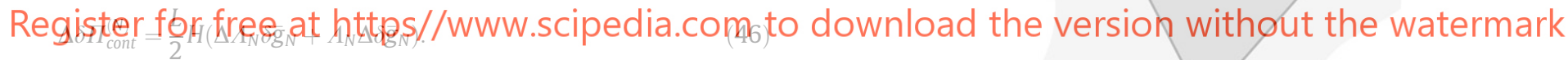

Its evaluation necessitates the linearization of the variation of the normal gap intensity

$$
\begin{aligned}
\Delta \delta \bar{g}_{N}= & -\bar{g}_{N}\left[\left(\mathbf{n} \cdot \frac{\partial \delta \mathbf{u}}{\partial t}\right)\left(\mathbf{n} \cdot \frac{\partial \Delta \mathbf{u}}{\partial t}\right)+\left(\mathbf{n} \cdot \frac{\partial \delta \mathbf{u}}{\partial t}\right)\left(\mathbf{t} \cdot \frac{\partial \Delta \mathbf{u}}{\partial n}\right)\right. \\
& \left.+\left(\mathbf{t} \cdot \frac{\partial \delta \mathbf{u}}{\partial n}\right)\left(\mathbf{n} \cdot \frac{\partial \Delta \mathbf{u}}{\partial t}\right)\right]
\end{aligned}
$$

(derived in the Appendix of the first part of this paper [14]), and the linearization of the discrete normal Lagrange multiplier $\Delta \Lambda_{N}$. Starting from Eq. (20) $)_{1}$ and using (21) $)_{1}$, the discrete normal Lagrange multiplier can alternatively be expressed with

$\Lambda_{N}=t_{N}+\frac{H}{2 \tau} \bar{g}_{N}$

Then its linearization yields

$\Delta \Lambda_{N}=\Delta t_{N}+\frac{H}{2 \tau} \Delta \bar{g}_{N}$,

where the linearization of the normal gap intensity has the same form as its variation (28):

$\Delta \bar{g}_{N}=\bar{g}_{N} \mathbf{n} \cdot \operatorname{grad}(\Delta \mathbf{u}) \cdot \mathbf{n}$.

All terms discussed so far depend upon the displacements of the vertices of a contact domain element. However, the linearization of the normal contact traction $\Delta t_{N}$ depends upon the displacements and the constitutive behavior of the adjacent "body" element (see Fig. 7), which involves the degrees of freedom of one additional finite element node in the interior of the contacting bodies (node 4).

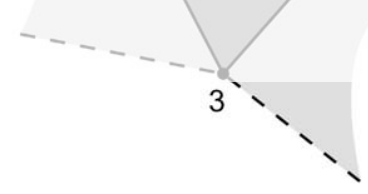

Fig. 7. Contact domain element with adjacent "body" element.

Starting from Eq. (10) $)_{1}$, the linearization of the normal contact traction yields

$$
\begin{aligned}
\Delta t_{N} & =\Delta \mathbf{n} \cdot \mathbf{P} \cdot \mathbf{N}+\mathbf{n} \cdot \Delta \mathbf{P} \cdot \mathbf{N} \\
& =-t_{T}(\mathbf{n} \cdot \operatorname{grad}(\Delta \mathbf{u}) \cdot \mathbf{t})+\mathbf{n} \cdot \Delta \mathbf{P} \cdot \mathbf{N},
\end{aligned}
$$

where

$\Delta \mathbf{n}=-(\mathbf{n} \cdot \operatorname{grad}(\Delta \mathbf{u}) \cdot \mathbf{t}) \mathbf{t}$,

derived in the Appendix of the first part of this paper [14], and Eq. $(10)_{2}$ have been used. The necessary linearization of the first PiolaKirchhoff stress tensor is detailed in the Appendix. Again, the first term in (51) only involves degrees of freedom of the contact domain element. Thus the linearization of the normal contact part may be split into two parts

$\Delta \delta \Pi_{\text {cont }}^{(N)}=\Delta \delta^{I} \Pi_{\text {cont }}^{(N)}+\Delta \delta^{I I} \Pi_{\text {cont }}^{(N)}$,

where part one ${ }^{(\mathrm{I})}$ only affects degrees of freedom within the contact domain element and part two ${ }^{(I I)}$ also affects the degrees of freedom in the adjacent "body" element. The two parts can then be specified with 
$\Delta \delta^{I} \Pi_{\text {cont }}^{(N)}=\frac{L}{2} H(-\underbrace{t_{T}(\mathbf{n} \cdot \operatorname{grad}(\Delta \mathbf{u}) \cdot \mathbf{t}) \delta \bar{g}_{N}}_{\text {non-sym }}+\frac{H}{2 \tau} \underbrace{\Delta \bar{g}_{N} \delta \bar{g}_{N}}_{\text {sym }}+\underbrace{\Lambda_{N} \Delta \delta \bar{g}_{N}}_{\text {sym }})$

and

$$
\Delta \delta^{I I} \Pi_{\text {cont }}^{(N)}=\frac{L}{2} H \underbrace{\mathbf{n} \cdot \Delta \mathbf{P} \cdot \mathbf{N} \delta \bar{g}_{N}}_{\text {non-sym }} .
$$

From Eqs. (54) and (55), it is obvious, that the resulting contact stiffness matrix becomes non-symmetric due to the linearization of the normal contact traction, which, in turn, stems from the added stabilization term in the variational constraint equations.

\subsubsection{Tangential contact - stick part}

Starting form Eq. (31), its linearization yields

$$
\Delta \delta \Pi_{\text {cont }}^{(T)}=\int_{D} \Delta \lambda_{T} \delta \bar{g}_{T} d D+\int_{D} \lambda_{T} \Delta \delta \bar{g}_{T} d D
$$

Performing the analytical integration, using Eq. (18) 1 , this expression can be written for one contact domain element with

$$
\Delta \delta \Pi_{\text {cont }}^{(T)}=\frac{L}{2} H\left(\Delta \Lambda_{T} \delta \bar{g}_{T}+\Lambda_{T} \Delta \delta \bar{g}_{T}\right) .
$$

Its evaluation necessitates the linearization of the variation of the tangential gap intensity
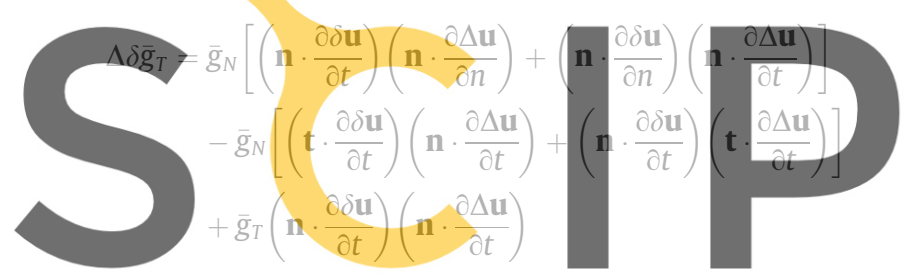

(derived in the first part of this paper [14]), and the linearization of

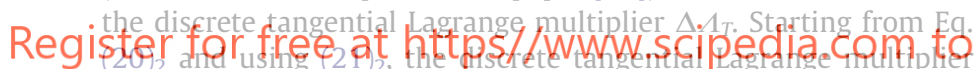
can alternatively be expressed with

$\Lambda_{T}=t_{T}+\frac{H}{2 \tau} \bar{g}_{T}$.

Then its linearization yields

$\Delta \Lambda_{T}=\Delta t_{T}+\frac{H}{2 \tau} \Delta \bar{g}_{T}$,

where the linearization of the tangential gap intensity has the same form as its variation (32):

$\Delta \bar{g}_{T}=\bar{g}_{N}(\mathbf{n} \cdot \operatorname{grad}(\Delta \mathbf{u}) \cdot \mathbf{t}+\mathbf{t} \cdot \operatorname{grad}(\Delta \mathbf{u}) \cdot \mathbf{n})+\bar{g}_{T} \mathbf{t} \cdot \operatorname{grad}(\Delta \mathbf{u}) \cdot \mathbf{t}$.

The linearization of the tangential contact traction $\Delta t_{T}$ depends upon the displacements and the constitutive behavior of the adjacent "body" element (see Fig. 7) and is given by the linearization of Eq. $(10)_{2}$

$\Delta t_{T}=\Delta \mathbf{t} \cdot \mathbf{P} \cdot \mathbf{N}+\mathbf{t} \cdot \Delta \mathbf{P} \cdot \mathbf{N}=t_{N}(\mathbf{n} \cdot \operatorname{grad}(\Delta \mathbf{u}) \cdot \mathbf{t})+\mathbf{t} \cdot \Delta \mathbf{P} \cdot \mathbf{N}$,

where

$\Delta \mathbf{t}=(\mathbf{n} \cdot \operatorname{grad}(\Delta \mathbf{u}) \cdot \mathbf{t}) \mathbf{n}$,

derived in the Appendix of the first part of this paper [14], and Eq. $(10)_{1}$ have been used. The linearization of the first Piola-Kirchhoff stress tensor is given in the Appendix. Similar to Section 4.6.1, the linearization for an active stick condition will be split into two parts

$\Delta \delta \Pi_{\text {cont }}^{(T)}=\Delta \delta^{I} \Pi_{\text {cont }}^{(T)}+\Delta \delta^{I I} \Pi_{\text {cont }}^{(T)}$, where part one $\mathrm{I}^{(\mathrm{I})}$ only affects degrees of freedom within the contact domain element and part two ${ }^{(\mathrm{II})}$ also affects the degrees of freedom in the adjacent "body" element. The two parts may then be specified with

$\Delta \delta^{I} \Pi_{\text {cont }}^{(T)}=\frac{L}{2} H(\underbrace{t_{N}(\mathbf{n} \cdot \operatorname{grad}(\Delta \mathbf{u}) \cdot \mathbf{t}) \delta \bar{g}_{T}}_{\text {non-sym }}+\frac{H}{2 \tau} \underbrace{\Delta \bar{g}_{T} \delta \bar{g}_{T}}_{\text {sym }}+\underbrace{\Lambda_{T} \Delta \delta \bar{g}_{T}}_{\text {sym }})$

and

$\Delta \delta^{I I} \Pi_{\text {cont }}^{(T)}=\frac{L}{2} H \underbrace{\mathbf{t} \cdot \Delta \mathbf{P} \cdot \mathbf{N} \delta \bar{g}_{T}}_{\text {non-sym }}$.

Due to the added stabilization term, again a non-symmetric contact stiffness matrix will arise.

4.6.3. Tangential contact - slip part

Starting form Eq. (35), its linearization yields

$\Delta \delta \Pi_{\text {cont }}^{(N \backslash T)}=\int_{D} \Delta \mathscr{T} \delta \bar{g}_{T} d D+\int_{D} \mathscr{T} \Delta \delta \bar{g}_{T} d D$.

Performing the analytical integration, using Eq. (18) $)_{1}$, this expression can be written for one contact domain element with

$\Delta \delta \Pi_{\text {cont }}^{(N) T}=\frac{L}{2} H\left(\Delta \mathscr{T} \delta \bar{g}_{T}+\mathscr{T} \Delta \delta \bar{g}_{T}\right)$.

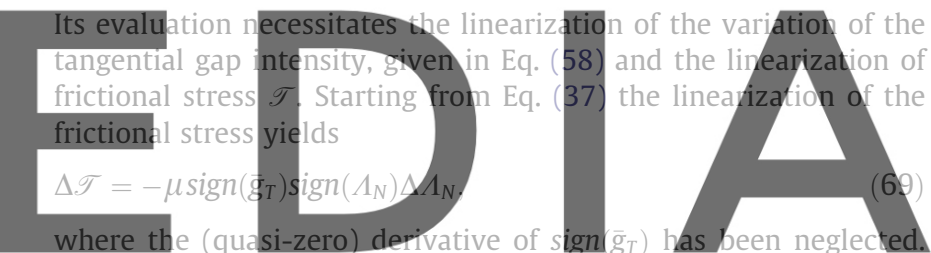

Similar to Sections 4.6.1 and 4.6.2, the linearization for an active slip condition will be split into two parts

download the version without the watermark $\Delta \delta \Pi_{\text {cont }}^{\text {con }}=\Delta \delta \Pi_{\text {cont }}+\Delta \delta \Pi_{\text {cont }}^{\text {ch }}$

where part one ${ }^{(1)}$ only affects degrees of freedom within the contact domain element and part two ${ }^{(I I)}$ also affects the degrees of freedom in the adjacent "body" element. Utilizing Eq. (37) and $\operatorname{sign}\left(\Lambda_{N}\right)=-1$, the two parts may then be specified with

$$
\begin{aligned}
\Delta \delta^{I} \Pi_{\text {cont }}^{(N \backslash T)}= & \frac{L}{2} H \mu \operatorname{sign}\left(\bar{g}_{T}\right) \\
& \times(-\underbrace{t_{T}(\mathbf{n} \cdot \operatorname{grad}(\Delta \mathbf{u}) \cdot \mathbf{t}) \delta \bar{g}_{T}}_{\text {non-sym }}+\frac{H}{2 \tau} \underbrace{\Delta \bar{g}_{N} \delta \bar{g}_{T}}_{\text {non-sym }}+\underbrace{\Lambda_{N} \Delta \delta \bar{g}_{T}}_{\text {sym }})
\end{aligned}
$$

and

$\Delta \delta^{I I} \Pi_{\text {cont }}^{(N \backslash T)}=\frac{L}{2} H \mu \operatorname{sign}\left(\bar{g}_{T}\right) \underbrace{\mathbf{n} \cdot \Delta \mathbf{P} \cdot \mathbf{N} \delta \bar{g}_{T}}_{\text {non-sym }}$.

The resulting contact stiffness matrix for the slip part is going to be non-symmetric as well, which is due to two reasons. On the one hand, this is induced by the non-symmetric Coulomb friction law (second term in Eq. (71)) and on the other hand due to the stabilization terms, that are only added to the variational constraint equations.

\subsection{Matrix notation}

In the following, a matrix notation will be introduced to compute the contact residuals as well as the resulting contact stiffness 
matrices for one contact domain element. As it has been shown in the previous section, the linearization of the contact contributions will also affect the degrees of freedom of the adjacent body element. Therefore a vector containing the variated nodal values

$\delta \mathbf{d}_{p}^{T}=\left(\delta \mathbf{d}_{1}, \delta \mathbf{d}_{2}, \delta \mathbf{d}_{3}, \delta \mathbf{d}_{4}\right)$

and a vector containing the incremental nodal displacements

$\Delta \mathbf{d}_{p}^{T}=\left(\Delta \mathbf{d}_{1}, \Delta \mathbf{d}_{2}, \Delta \mathbf{d}_{3}, \Delta \mathbf{d}_{4}\right)$

is defined, based on the four vertex/nodes indicated in Fig. 7. Furthermore, the following matrices will be defined:

$\mathbf{N}_{n}=\left(\begin{array}{c}\left(g_{N}\right)_{3} \frac{\partial N_{1}}{\partial n} \mathbf{n} \\ \left(g_{N}\right)_{3} \frac{\partial N_{2}}{\partial n} \mathbf{n} \\ \left(g_{N}\right)_{3} \frac{\partial N_{3}}{\partial n} \mathbf{n} \\ \mathbf{0}\end{array}\right) ; \quad \mathbf{N}_{t}=\left(\begin{array}{c}\frac{\partial N_{1}}{\partial t} \mathbf{n} \\ \frac{\partial N_{2}}{\partial t} \mathbf{n} \\ \frac{\partial N_{3}}{\partial t} \mathbf{n} \\ \mathbf{0}\end{array}\right)$

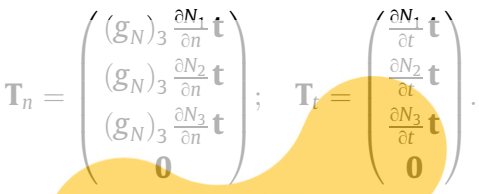

The matrices $\mathbf{N}_{\sigma P}$ and $\mathbf{T}_{\sigma P}$ to compute

$\mathbf{n} \cdot \Delta \mathbf{P} \cdot \mathbf{N}=\mathbf{N}_{\sigma P}^{T} \Delta \mathbf{d}_{p}$ and $\mathbf{t} \cdot \Delta \mathbf{P} \cdot \mathbf{N}=\mathbf{T}_{\sigma P}^{T} \Delta \mathbf{d}_{p}$,

are given with

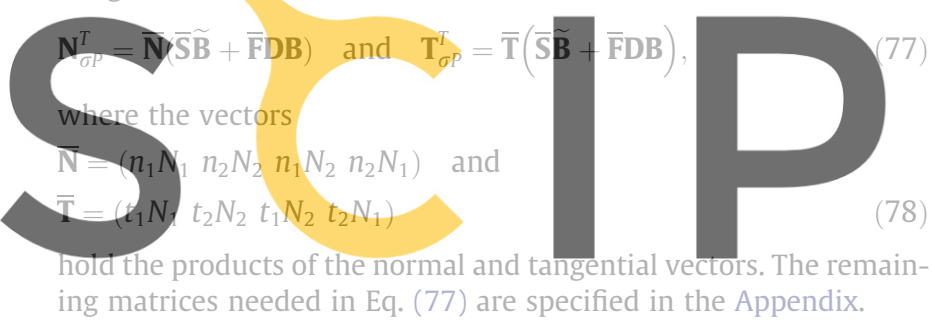

\section{Register for free at https//www.scipedia.com to}

With the definitions of the matrices in (75) and (77), the normal part of the contact virtual work done by one contact patch (Eq. (29)) can be written in matrix formulation $\delta \mathbf{d}^{T} \mathbf{G}_{\text {cont }}^{(N)}$ with the elemental contact residual

$\mathbf{G}_{\text {cont }}^{(N)}=\frac{L}{2} \Lambda_{N} \mathbf{N}_{n}$

Furthermore the linearization of the normal contact contributions (54) and (55) leads to

$\Delta \delta^{I} \Pi_{\text {cont }}^{(N)}+\Delta \delta^{I I} \Pi_{\text {cont }}^{(N)}=\delta \mathbf{d}^{T}\left({ }^{I} \mathbf{K}_{\text {cont }}^{(N)}+{ }^{I I} \mathbf{K}_{\text {cont }}^{(N)}\right) \Delta \mathbf{d}$,

with the elemental contact stiffness matrices

${ }^{I} \mathbf{K}_{\text {cont }}^{(N)}=-\frac{L}{2} \Lambda_{N}\left(\left(g_{N}\right)_{3} \mathbf{N}_{t} \mathbf{N}_{t}^{T}+\mathbf{T}_{n} \mathbf{N}_{t}^{T}+\mathbf{N}_{t} \mathbf{T}_{n}^{T}\right)+\frac{L}{4 \tau} \mathbf{N}_{n} \mathbf{N}_{n}^{T}$

$$
-\frac{L}{2} t_{T} \mathbf{N}_{n} \mathbf{N}_{t}^{T}
$$

and

${ }^{I I} \mathbf{K}_{\text {cont }}^{(N)}=\frac{L}{2} \mathbf{N}_{n} \mathbf{N}_{\sigma P}^{T}$.

\subsubsection{Tangential contact - stick part}

The stick part of the contact virtual work (Eq. (33)) is given in matrix notation $\delta \mathbf{d}^{T} \mathbf{G}_{\text {cont }}^{(T)}$ with the elemental contact residual

$\mathbf{G}_{\text {cont }}^{(T)}=\frac{L}{2} \Lambda_{T}\left(\left(g_{N}\right)_{3} \mathbf{N}_{t}+\mathbf{T}_{n}+\left(g_{T}\right)_{3} \mathbf{T}_{t}\right)$.

The matrix formulation of the linearization of the stick contributions (65) and (66) reads
$\Delta \delta^{I} \Pi_{\text {cont }}^{(T)}+\Delta \delta^{I I} \Pi_{\text {cont }}^{(T)}=\delta \mathbf{d}^{T}\left({ }^{I} \mathbf{K}_{\text {cont }}^{(T)}+{ }^{I I} \mathbf{K}_{\text {cont }}^{(T)}\right) \Delta \mathbf{d}$,

with the elemental contact stiffness matrices

$$
\begin{aligned}
{ }^{I} \mathbf{K}_{\text {cont } t}^{(T)}= & \frac{L}{2} \Lambda_{T}\left(\mathbf{N}_{t} \mathbf{N}_{n}^{T}+\mathbf{N}_{n} \mathbf{N}_{t}^{T}+\left(g_{T}\right)_{3} \mathbf{N}_{t} \mathbf{N}_{t}^{T}-\left(g_{N}\right)_{3} \mathbf{T}_{t} \mathbf{N}_{t}^{T}-\left(g_{N}\right)_{3} \mathbf{N}_{t} \mathbf{T}_{t}^{T}\right) \\
& +\frac{L}{4 \tau}\left(\left(g_{N}\right)_{3} \mathbf{N}_{t}+\mathbf{T}_{n}+\left(g_{T}\right)_{3} \mathbf{T}_{t}\right)\left(\left(g_{N}\right)_{3} \mathbf{N}_{t}+\mathbf{T}_{n}\right. \\
& \left.+\left(g_{T}\right)_{3} \mathbf{T}_{t}\right)^{T}+\frac{L}{2} t_{N}\left(\left(g_{N}\right)_{3} \mathbf{N}_{t}+\mathbf{T}_{n}+\left(g_{T}\right)_{3} \mathbf{T}_{t}\right) \mathbf{N}_{t}^{T}
\end{aligned}
$$

and

${ }^{I I} \mathbf{K}_{\text {cont }}^{(T)}=\frac{L}{2}\left(\left(g_{N}\right)_{3} \mathbf{N}_{t}+\mathbf{T}_{n}+\left(g_{T}\right)_{3} \mathbf{T}_{t}\right) \mathbf{T}_{\sigma P}^{T}$

\subsubsection{Tangential contact - slip part}

The slip part of the contact virtual work ( $\mathrm{Eq} .(36)$ ) is given in matrix notation $\delta \mathbf{d}^{T} \mathbf{G}_{\text {cont }}^{(N T)}$ with the elemental contact residual

\section{$\mathbf{G}_{\text {cont }}^{(N \backslash T)}=\frac{L}{2} \Lambda_{N} \mu \operatorname{sign}\left(\bar{g}_{T}\right)\left(\left(g_{N}\right)_{3} \mathbf{N}_{t}+\mathbf{T}_{n}+\left(g_{T}\right)_{3} \mathbf{T}_{t}\right)$.}

The matrix formulation of the linearization of the slip contributions (71) and (72) reads

$\Delta \delta^{I} \Pi_{\text {cont }}^{(N \backslash T)}+\Delta \delta^{I I} \Pi_{\text {cont }}^{(N \backslash T)}=\delta \mathbf{d}^{T}\left({ }^{I} \mathbf{K}_{\text {cont }}^{(N \backslash T)}+{ }^{I I} \mathbf{K}_{\text {cont }}^{(N \backslash T)}\right) \Delta \mathbf{d}$

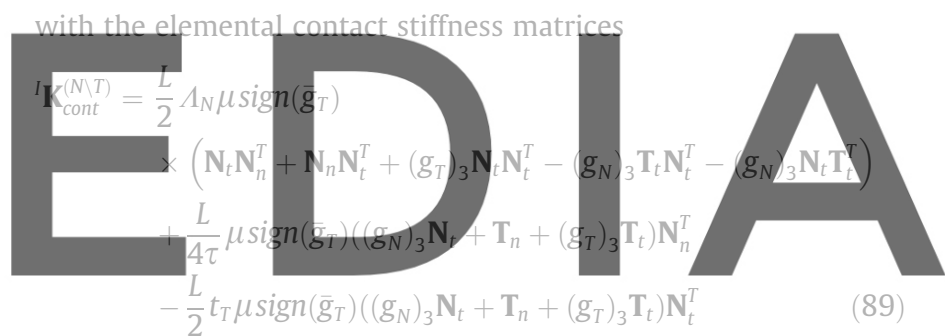

\section{download the version without the watermark}

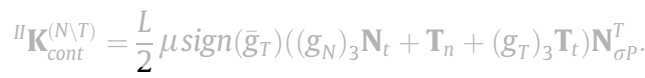

Remark 4.1. Looking at the contact stiffness matrices derived in this section, it becomes obvious, that they all exhibit non-symmetric parts. This stems from the introduction of the additional (stabilization) terms only in the constrained variational equations (9). Although Heintz and Hansbo [8] have proposed a consistent symmetric version of stabilization, the authors have decided to add the stabilization terms only in the constrained equations, as the Coulomb's friction law makes the problem non-symmetric anyway.

\section{Active set strategy - technical aspects}

The contact formulation presented so far is based on the assumption that the active set of contact domain elements being in contact at the end of one specific time step is known "a priori". This includes the active normal contact condition as well as the frictional conditions, distinguishing also between stick or slip. In the first part of this paper [14] a predictive active set strategy, based on so-called active constraint indicators is presented. In the following, some important technical details about the important extrapolation procedure are discussed.

\subsection{Prediction of effective gaps}

For the proposed active set strategy, the so-called effective normal and tangential gaps 
$g_{N}^{e f f .} \equiv\left(g_{N}\right)_{3}+2 \tau t_{N}$,

$g_{T}^{\text {eff. }} \equiv\left(g_{T}\right)_{3}+2 \tau t_{T}$

and suitable (displacement based) active constraint indicators

$D^{(p)} \subset D^{(N)} \Longleftrightarrow \beta_{N} \stackrel{\text { def }}{=} g_{N}^{\text {eff. }}<0 \rightarrow$ active normal contact,

$D^{(p)} \subset D^{(T)} \Longleftrightarrow \beta_{T} \stackrel{\text { def }}{=}\left|g_{T}^{\text {eff. }}\right|-\mu\left|g_{N}^{\text {eff. }}\right|<0 \rightarrow$ active stick,

are defined for every contact domain element. In order to predict the active contact/friction element set for the next time step, a first order approximation of these indicators is performed on basis of the evolution of the effective normal and tangential gaps in previous time steps:

$\tilde{g}_{N / T}^{\text {eff. }}=\left(g_{N / T}^{\text {eff. }}\right)_{n}+\frac{\Delta t_{n+1}}{\Delta t_{n}} \Delta g_{N / T}^{\text {eff. }}$

with

$\Delta g_{N / T}^{\text {eff. }}=\left(g_{N / T}^{\text {eff. }}\right)_{n}-\left(g_{N / T}^{\text {eff. }}\right)_{n-1}$,

where the subscripts $(\cdot)_{n}$ and $(\cdot)_{n-1}$ refer to the end of the time steps $n$ and $n-1$. Therefore, the effective normal and tangential gaps of the previous time steps need to be appropriately stored.

\subsection{History of effective gaps}

Recording the history of the effective gaps is by no means a trivial task. The effective gaps are defined for every contact domain element, providing information about this specific set of finite element nodes on the contacting boundaries. One specific feature of the presented contact strategy is the reconstruction of the contact domain mesh, whenever the relative motion of the contacting surfaces exceeds some tolerances. In fact, contact problems involving large relative sliding require a reconnection of boundary nodes very frequently, sometimes even after every time step. Consequently it is not possible to just store the effective gaps within the contact domain element, as this element may not exist any-

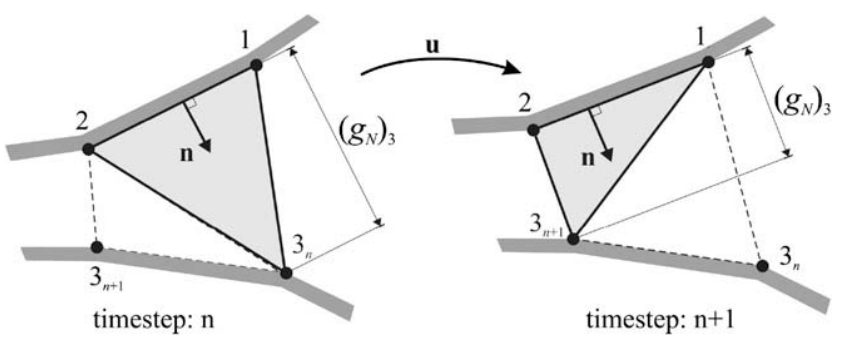

Fig. 8. Change of connectivity - influence on gaps. more in the ensuing time step. Furthermore, the values of the effective gaps strongly depend on the connectivity of the contact domain mesh. If the connectivity changes from one time step to the other (see Fig. 8) it might happen, that the normal gap $\left(g_{N}\right)_{3}$ of the vertex node 3 , as a main part for the calculation of the effective normal gap, alters erratically. The effective gaps would not vary smoothly and the extrapolation of these values would not be beneficial. To overcome this, a different procedure for keeping track of the evolution of the effective gaps is necessary.

For the presented contact algorithm the following approach is used. Instead of storing the effective gaps themselves, the placement of the finite element nodes on the contact boundary, as well as the normal and tangential surface tractions $\left(t_{N}, t_{T}\right)$ at the boundary of the "body" elements, are stored for the previous time steps $n$ and $n-1$. Based on the connectivity of a contact domain element in the considered time step, the effective gaps are calculated retrospective.

In Fig. 9 the placement of a contact domain element is shown at the beginning of three consecutive time steps, thus representing in each case the reference configuration. Based on the connectivity of the considered contact domain element at the present time step (nodes $1-2-3$ ), the fictive initial normal gaps $\left(g_{N}^{(0)}\right)_{3}^{*}$ at the beginning of the two previous time steps are calculated with

$$
\begin{aligned}
& \left.\left(g_{N}^{(0)}\right)_{3}^{*}\right|_{n-1}=\left[\left(\mathbf{x}_{3^{*}}\right)_{n-2}-\left(\mathbf{x}_{2}\right)_{n-2}\right] \cdot \mathbf{N}_{n-1} \text { and } \\
& \left.\left(g_{N}^{(0)}\right)_{3}^{*}\right|_{n}=\left[\left(\mathbf{x}_{3^{*}}\right)_{n-1}-\left(\mathbf{x}_{2}\right)_{n-1}\right] \cdot \mathbf{N}_{n} .
\end{aligned}
$$

With this, the normal and tangential gaps $\left(g_{N}\right)_{3}$ and $\left(g_{T}\right)_{3}$ (see Eq. (26)) are evaluated for the two previous time steps

$$
\begin{aligned}
\left.\left(g_{N}\right)_{3}\right|_{n-1}= & \left.\left(g_{N}^{(0)}\right)_{3}^{*}\right|_{n-1} \mathbf{n}_{n-1} \cdot \mathbf{N}_{n-1} \\
& \quad+\mathbf{n}_{n-1} \cdot \sum_{I=1}^{3}\left(\left.\left(g_{N}^{(0)}\right)_{3}^{*}\right|_{n-1} \frac{\partial N_{I}}{\partial N_{n-1}}\right)\left(\mathbf{d}_{I}\right)_{n-1}, \\
\left.\left(g_{T}\right)_{3}\right|_{n-1}=\left.\left(g_{N}^{(0)}\right)_{3}^{*}\right|_{n-1} \mathbf{t}_{n-1} \cdot \mathbf{N}_{n-1} & \\
& +\mathbf{t}_{n-1} \cdot \sum_{I=1}^{3}\left(\left.\left(g_{N}^{(0)}\right)_{3}^{*}\right|_{n-1} \frac{\partial N_{I}}{\partial N_{n-1}}\right)\left(\mathbf{d}_{I}\right)_{n-1}
\end{aligned}
$$

and

$$
\begin{aligned}
& \left.\left(g_{N}\right)_{3}\right|_{n}=\left.\left(g_{N}^{(0)}\right)_{3}^{*}\right|_{n} \mathbf{n}_{n} \cdot \mathbf{N}_{n}+\mathbf{n}_{n} \cdot \sum_{I=1}^{3}\left(\left.\left(g_{N}^{(0)}\right)_{3}^{*}\right|_{n} \frac{\partial N_{I}}{\partial N_{n}}\right)\left(\mathbf{d}_{I}\right)_{n}, \\
& \left.\left(g_{T}\right)_{3}\right|_{n}=\left.\left(g_{N}^{(0)}\right)_{3}^{*}\right|_{n} \mathbf{t}_{n} \cdot \mathbf{N}_{n}+\mathbf{t}_{n} \cdot \sum_{I=1}^{3}\left(\left.\left(g_{N}^{(0)}\right)_{3}^{*}\right|_{n} \frac{\partial N_{I}}{\partial N_{n}}\right)\left(\mathbf{d}_{I}\right)_{n}
\end{aligned}
$$

with

$\left(\mathbf{d}_{I}\right)_{n-1}=\left(\mathbf{x}_{I}\right)_{n-1}-\left(\mathbf{x}_{I}\right)_{n-2} \quad$ and $\quad\left(\mathbf{d}_{I}\right)_{n}=\left(\mathbf{x}_{I}\right)_{n}-\left(\mathbf{x}_{I}\right)_{n-1}$.

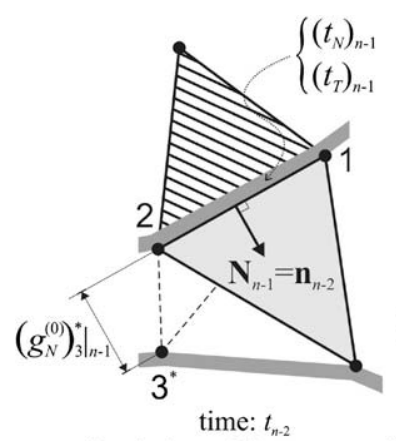

(beginning of time step: $n-1$ )

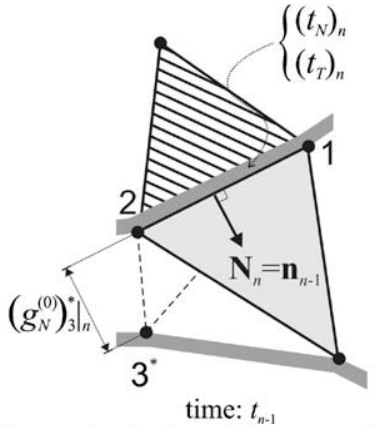

(beginning of time step: $n$ )

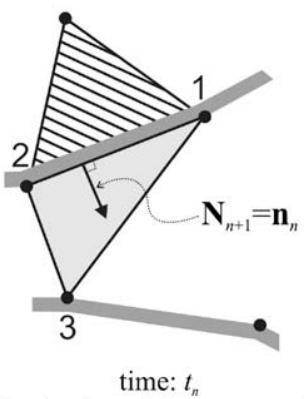

(beginning of time step: $n+1$ )

Fig. 9. Reference configuration of different time steps 
Together with the stored normal and tangential tractions $\left(t_{N}, t_{T}\right)$, at the boundary of the adjacent "body" element (see Fig. 9), the effective gaps for the previous time steps can be calculated using Eq. (91). Having these quantities at hand, the extrapolation of the effective normal and tangential gaps can be performed as described in the first part of this paper [14], Section 5.5.

\section{Numerical examples}

The performance of the presented contact strategy is evaluated by means of a set of numerical examples. All the examples are computed under the assumption of a plane strain condition with a unit thickness of 1.0, using a compressible neo-Hookean, hyperelastic material [2]. For the element-wise constant stability parameter $\tau$ (in Eqs. (9) and (20)) the relation

$\tau=\frac{\alpha_{\text {stab }}}{E_{\min }} L$

is used, where $E_{\min }$ is the minimal Young's modulus of the contacting bodies, $L$ is the base-side length of the contact domain element in the previous configuration (see Fig. 2) and $\alpha_{\text {stab }}$ is a dimensionless, user defined, parameter which is independent of the meshsize. The relation in Eq. (99) stems from the fact that the dimension of the stabilization parameter $\tau$ needs to be $\left[\mathrm{m}^{3} / \mathrm{N}\right]$ in order to give the correct dimensions within the variational constraint equations (9). Furthermore, a suitable value of the stabilization parameter $\tau$ is governed by the present stresses in the contact interface, which in turn depend upon the Young's modulus. Finally the stabilization parameter $\tau$ needs to be adjusted with the characteristic size of a contact domain element.

For the static analyses performed, the inertia term Mä in Eq. (39) drops out of the effective structural equation and therefore the non-linear static problem can be solved without using the described time integration scheme. Dealing with dynamic problems, the Generalized- $\alpha$ time integration scheme, described very briefly in Section 4.5 is utilized, where the so-called spectral radius $\rho_{\infty}$ defines the values of the introduced interpolation and Newmark parameters:

$\alpha_{m}=\frac{2 \rho_{\infty}-1}{\rho_{\infty}+1} ; \quad \alpha_{f}=\frac{\rho_{\infty}}{\rho_{\infty}+1} ; \quad \beta=\frac{1}{4}\left(1-\alpha_{m}+\alpha_{f}\right)^{2} ;$

$\gamma=\frac{1}{2}-\alpha_{m}+\alpha_{f}$

\subsection{Contact patch test}

A contact formulation should be able to exactly transmit a spatially constant stress field from one body to another along an arbitrary non-conforming contact surface. This ability will be checked by the so-called contact patch test [11]. Different patch test setups have been proposed in the literature and the authors have decided to use one, recently considered in $[5,4]$. The configuration of the problem is shown in Fig. 10a. An elastic body is pressed with a constant pressure of $p=100.0\left[\mathrm{~N} / \mathrm{mm}^{2}\right]$ onto another elastic body, assuming a frictionless contact behavior. The punch, as well as the foundation are taken as linear elastic isotropic media with the same material properties. Fig. 10b depicts the finite element discretization of the two bodies, using very different mesh densities in each of them.

The numerical results are shown in Fig. 11 in terms of the consistent nodal forces, for different contact pairing strategies. Approximating the contact domain with a full set of non-overlapping patches, as proposed in this paper, the contact patch test is properly passed and the resulting consistent nodal forces coincide with the exact ones (see Fig. 11a). It is worth noting that this result is totally independent of the utilized value of the stabilization parameter $\alpha_{\text {stab }}$. However, in order to demonstrate the necessity of having a complete approximation of the contact domain, additional pairing strategies, reproducing typical pairings used in node-to-segment approaches are analyzed. Fig. 11b and c depicts contact pairing scenarios that would correspond to a single-pass node-to-segment approach, assuming the top, respectively the bottom surface to be the slave surface. A contact pairing scenario mimicking a two-pass node-to-segment approach is shown in Fig. 11d. The resulting consistent nodal forces for these pairing scenarios are given in Fig. $11 \mathrm{~b}-\mathrm{d}$, where $\alpha_{\mathrm{stab}}=0.1$ has been used. Obviously, none of them passes the contact patch test. It follows that a key issue of the proposed contact domain method is the usage of a full set of non-overlapping patches, including contact domain elements that might be distorted (having its normal projection of the single vertex node outside of the base-side).

Remark 6.1. The usage of distorted contact domain elements is somehow contradictory to the widely used closest-point projection procedure and one could wonder about the consequences, once the contact interface is not a straight line as in the discussed contact patch test. However, Fig. 12 demonstrates that the usage of a patch of contact domain elements will lead in summary to the correct projection. Working only with the contact domain element 1 , node A will be forced to lie onto the extension of its base-side (to some point A1), and similarly utilizing only the contact domain element 2 , node A will be projected to some point A2 of the corresponding extension of its base-side. If the contact domain is made of both elements 1 and 2, node $A$ will be forced to lie on the intersection of both base-sides, which is node $B$, which in turn is the closestpoint projection of node A onto the surface of the other contacting body. In summary, the local, element-wise definition of the geometric gaps (possibly violating the closest-point methodology), leads to a globally correct enforcement of the geometrical contact constraints, under the assumption of using a full set of non-overlapping patches. (a)

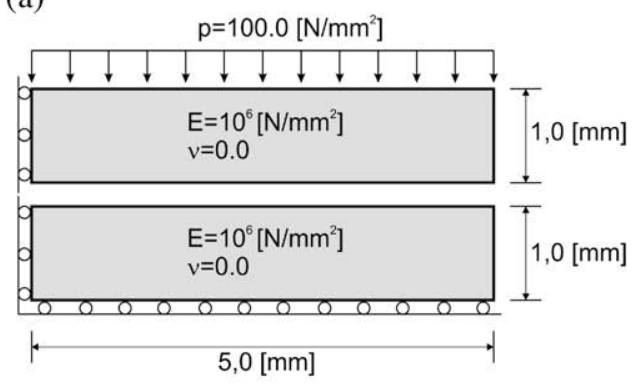

(b)

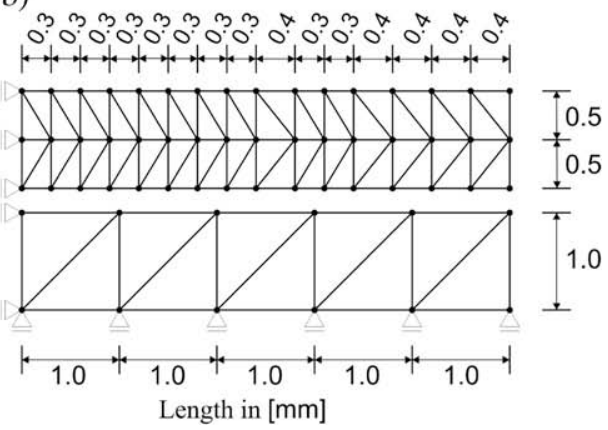

Fig. 10. Contact patch test: (a) setup; (b) finite element mesh. 
(a)

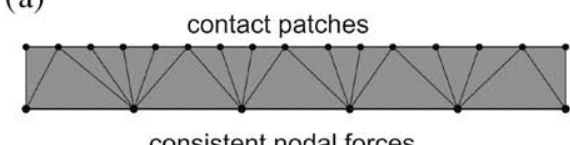

consistent nodal forces

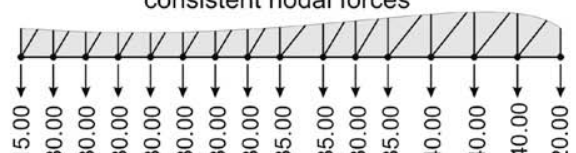

(b)

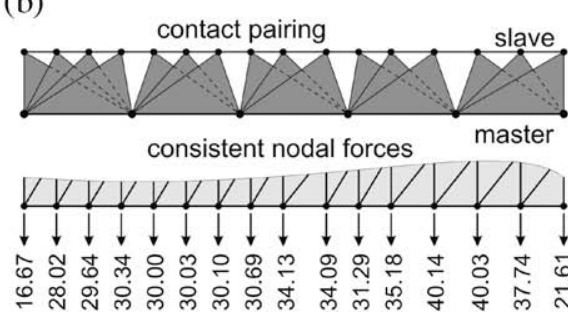

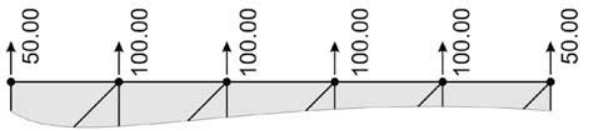

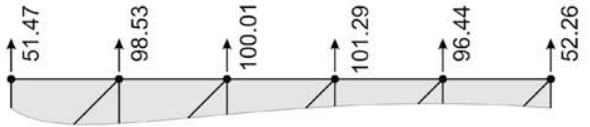

(c)

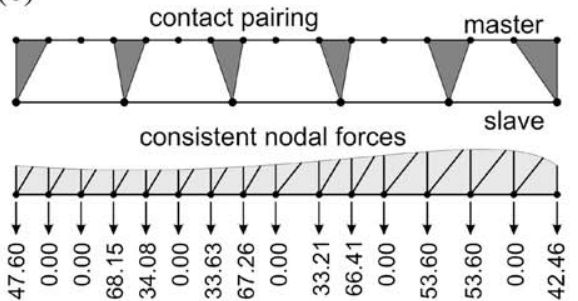

(d)

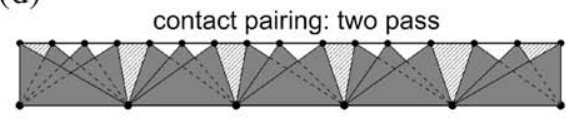

consistent nodal forces
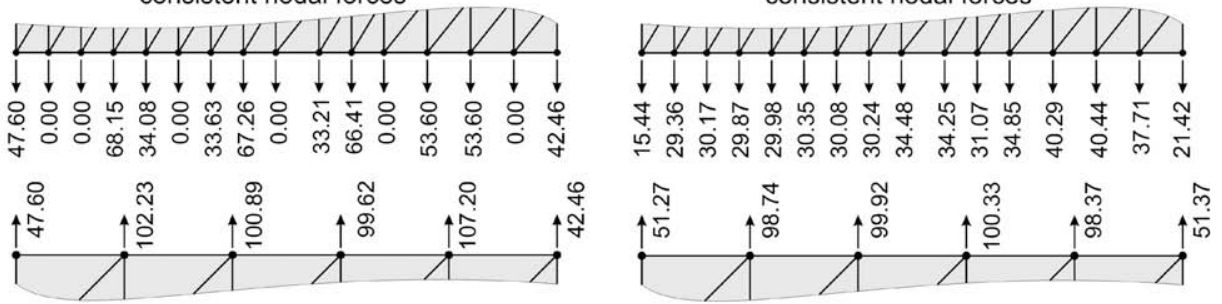

Fig. 11. Consistent nodal force distribution: (a) CDM/exact; (b) top surface as slave; (c) bottom surface as slave; (d) two-pass.

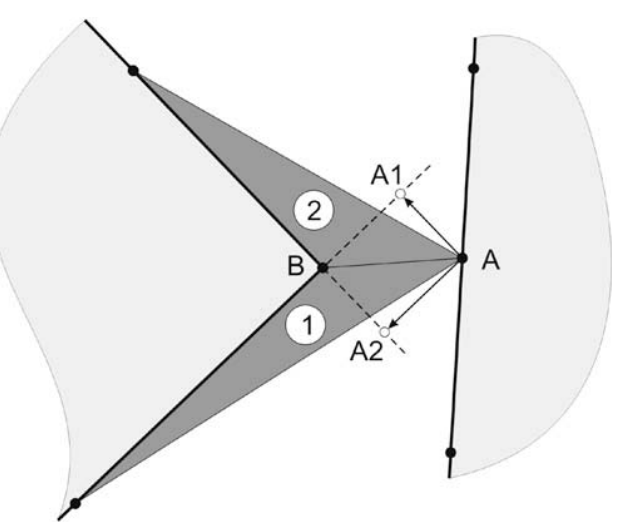

Fig. 12. Corner scenario.

\subsection{Frictional Hertzian contact}

To investigate the accuracy of the proposed contact formulation a cylinder on cylinder Hertzian contact problem is analyzed. The underlying analytical solution dates back to the work of Hertz [9], which assumes infinitesimal small deformations. In order to compare the results with the proposed large deformation contact strategy a very little load is applied. The geometric setup as well as the utilized finite element discretization is shown in Fig. 13. Two elastic half cylinders $\left(E=200.0\left[\mathrm{~N} / \mathrm{mm}^{2}\right], v=0.3\right)$ are first pressed together with a compressive normal force $P=\int p(x) d s=10.0[\mathrm{~N}]$. Subsequently a tangential force $Q=\int q(x) d s=1.0[\mathrm{~N}]$ is applied which causes tangential contact tractions in the contact zone. The width $b$, being the half of the contact zone, can then be calculated analytically with

$b=2 \sqrt{P R \frac{1-v^{2}}{\pi E}}=0.6808[\mathrm{~mm}]$,
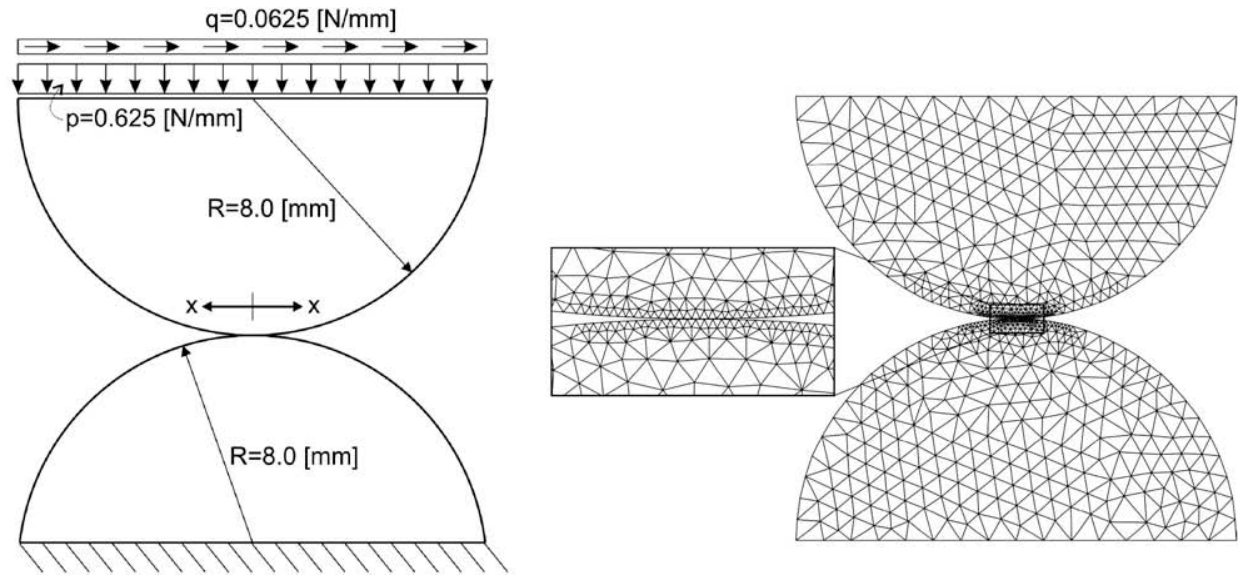

Fig. 13. Cylinder on cylinder Hertzian contact problem and the (rough) discretization. 
where $R$ is the radius of the two cylinders. The normal pressure distribution in the contact zone is given with

$\sigma_{n}=\sigma_{n}^{\max } \sqrt{1-\left(\frac{x}{b}\right)^{2}}$

where $\sigma_{n}^{\max }$ is the maximum normal pressure computed via

$\sigma_{n}^{\max }=\sqrt{\frac{P E}{\pi\left(1-v^{2}\right) R}}=9.351\left[\mathrm{~N} / \mathrm{mm}^{2}\right]$.

For the frictional contact part a Coulomb friction coefficient of $\mu=0.2$ is assumed. Increasing the applied tangential forces $q$ from zero to the maximal value, micro-slip evolves starting from the edges of the contact zone. Therefore the contact zone is subdivided into a stick part $|x| \leqslant|c|$ and two areas of slip $|c| \leqslant|x| \leqslant|b|$. The size of the stick region is given by

$c=b \sqrt{1-\frac{Q}{\mu P}}=0.481[\mathrm{~mm}]$.

Thus the distribution of the tangential contact tractions can be calculated as follows:

$\sigma_{t}(x)=\frac{\mu \sigma_{n}^{\max }}{b}\left(\sqrt{b^{2}-x^{2}}-\sqrt{c^{2}-x^{2}}\right):|x| \leqslant|c|$,

$\sigma_{t}(x)=\mu \sigma_{n}(x):|c| \leqslant|x| \leqslant|b|$.

In Fig. 14 the numerical solution is plotted against the analytical solution, where the fine dashed line indicates the maximum tangential traction $\mu \sigma_{n}$. The abrupt change in the tangential traction stems from the transition from stick to slip. The shown numerical results are calculated using a stabilization parameter $\alpha_{\text {stab }}=0.5$ (see Eq. (99)). It should be mentioned, that the influence of the stabilization parameter in this example is not significant. A variation of this parameter between $\alpha_{\text {stab }}=[0.1 \rightarrow 5.0]$ produces qualitatively and quantitatively comparable results, as the deformations are very small and therefore the contact is very well imposed regardless of the utilized stabilization parameter. When the geometrical contact condition is very well imposed, the normal and tangential gaps defined in Eq. (26) tend to zero and thus the discrete normal and tangential Lagrange multipliers (see Eq. (20)) have the property of representing the normal and tangential surface tractions at the boundaries of the contacting bodies $\left(\Lambda_{N} \approx t_{N}\right.$ and $\left.\Lambda_{T} \approx t_{T}\right)$. Therefore, the discrete Lagrange multipliers are utilized to check the normal and tangential contact tractions against the exact analytical solution. As the introduced Lagrange multipliers are element-wise constant values, they will be discontinuous at the boundary nodes. Therefore nodal values of the Lagrange multipliers are calculated, using the weighted nodal sum of the discrete, constant elemental values of the introduced Lagrange multipliers at the adjacent contact elements to one contacting node:

$\Lambda^{\text {node }}=\frac{\sum_{p=1}^{n_{p}} \Lambda^{(p)} L^{(p)}}{\sum_{p=1}^{n_{p}} L^{(p)}}$.

Herein $\Lambda^{\text {node }}$ depicts the average nodal Lagrange multiplier, $n_{p}$ is the number of adjacent active contact domain elements to this specific boundary node and $\Lambda^{(p)}$ and $L^{(p)}$ are the elemental constant Lagrange multiplier and the length of the base-side of the considered contact domain element, respectively. In general one would weigh the elemental Lagrange multipliers with the area of the element, but as the elemental height $H^{(p)}$ denotes a measure of the actual gap, this tends to zero for active contact elements and thus has no significant meaning. Therefore only the length $L^{(p)}$ of a contact element is taken into account.

Looking at the results plotted in Fig. 14 one can observe that the contact tractions are quite well represented by the developed contact algorithm. Due to the non-smoothness of the frictional traction field at the stick slip boundary, some oscillations in the numerical solution for the frictional tractions can be observed. Using a finer discretization, these oscillations diminish considerably (see Fig. 15).

\subsection{Beam bending}

This example was presented by Puso and Laursen $[15,16]$ to demonstrate the locking free behavior of their proposed mortar segment-to-segment contact strategy. Initially, two equally sized beams $(10.0 \times 1.0)$ are pressed together by a constant pressure $p=0.1$ and then bent applying a moment to the end of the two beams. The initial geometry, the material data, the boundary conditions and the applied loads are given in Fig. 16.

As a reference solution this problem is first analyzed assuming one homogeneous beam, such that the deformations are conforming across the interface. In Fig. 17a the initial and deformed configuration for the homogeneous beam is shown as well as its triangulation, utilizing a rough mesh. Now the two beams are discretized separately, using dissimilar triangulations along the frictionless, sliding contact interface. Fig. $17 \mathrm{~b}-\mathrm{d}$ shows the utilized

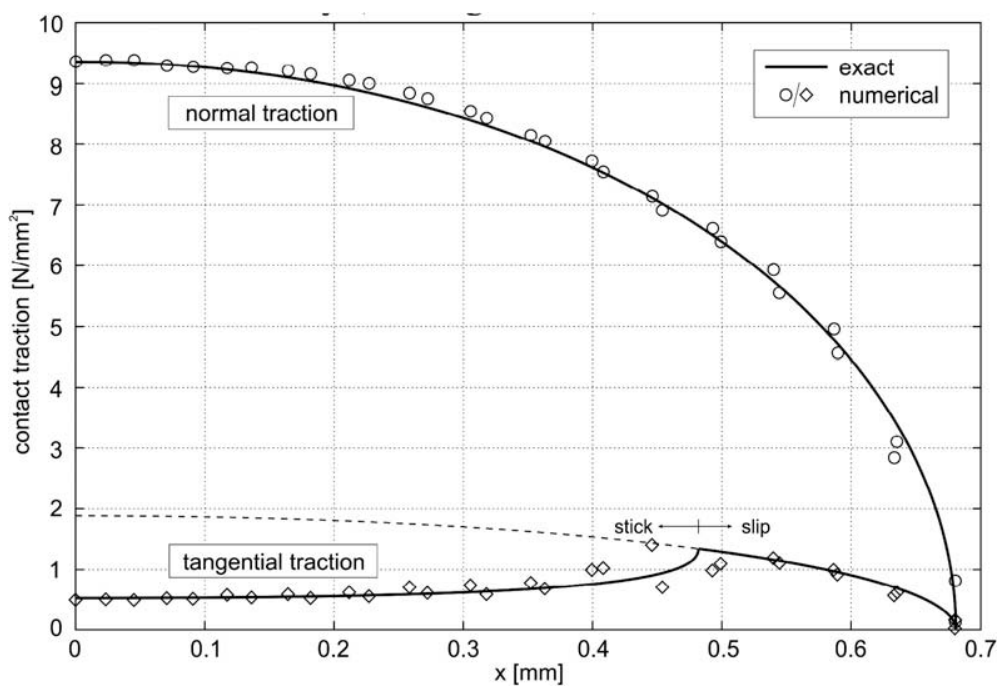

Fig. 14. Computed nodal contact traction for frictional cylinder on cylinder contact problem; coarse mesh. 


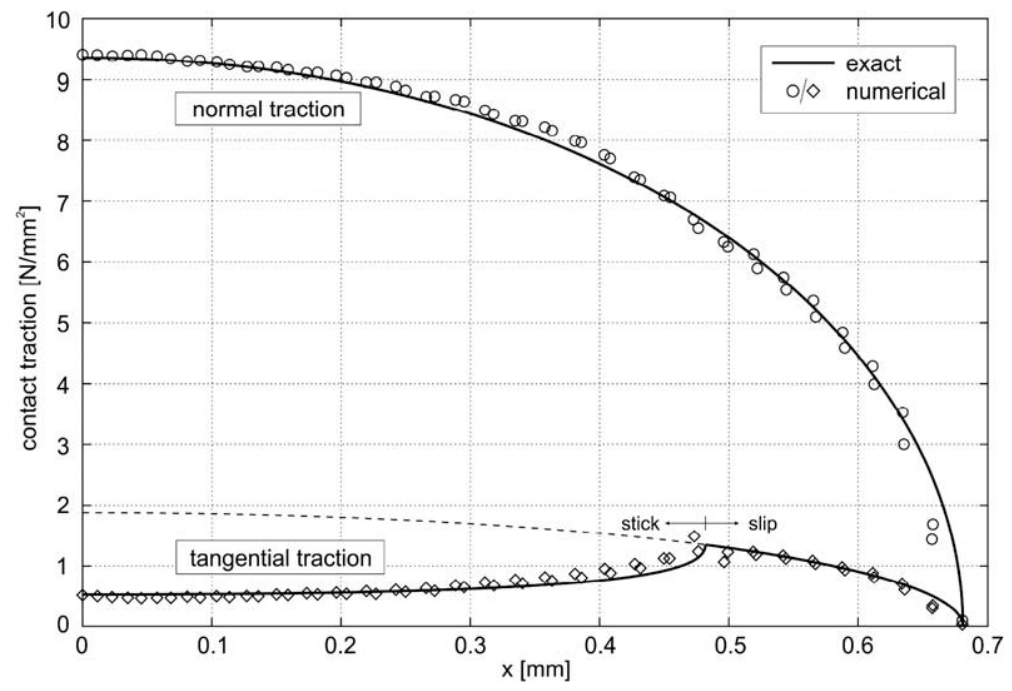

Fig. 15. Computed nodal contact traction for frictional cylinder on cylinder contact problem; finer mesh.

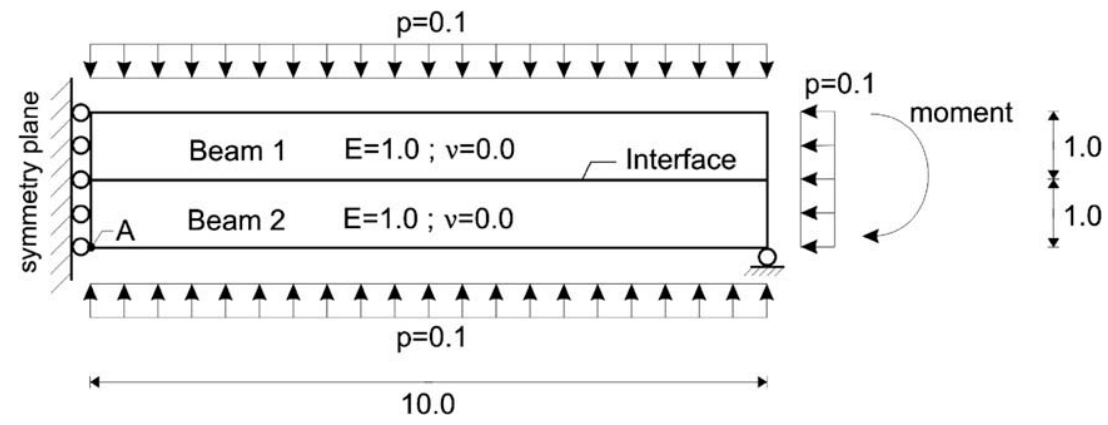

Fig. 16. Beam bending: Geometry, material data, boundary conditions and applied loads.

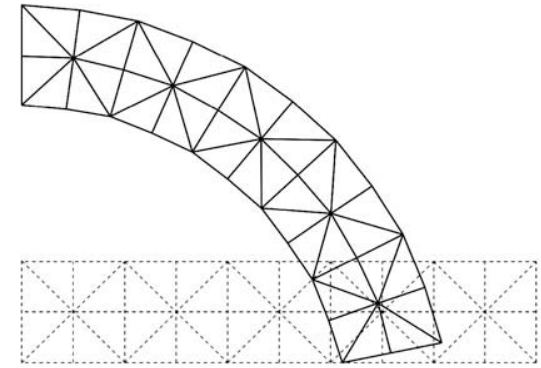

(a) conforming

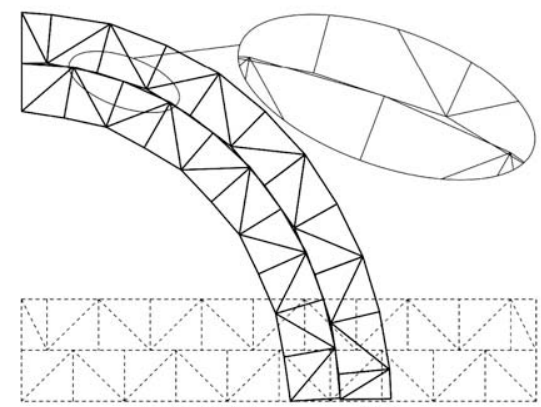

(c) CDM, $\alpha_{\text {stab }}=0.5$

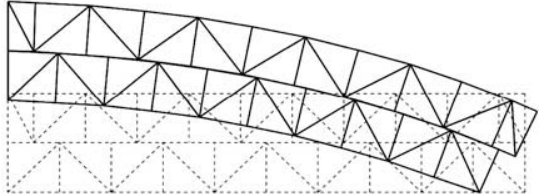

(b) CDM, $\alpha_{\text {stab }}=0.01$

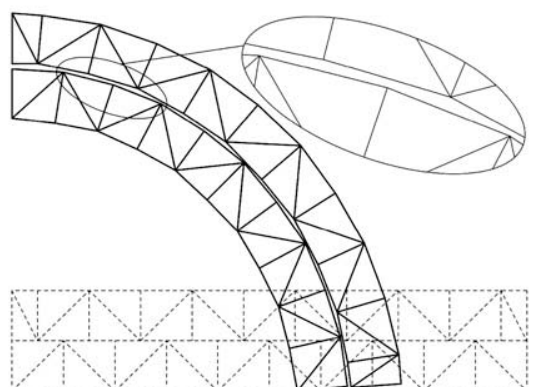

(d) CDM, $\alpha_{\text {stab }}=10.0$

Fig. 17. Initial and deformed configuration (rough mesh): (a) homogeneous discretization without contact; (b)-(d) sandwiched beams using the contact domain method with $\alpha_{\text {stab }}=0.01 / 0.5 / 10.0$. 
finite element meshes and the results with the presented "contact domain method (CDM)" using three different values of the stabilization parameter $\alpha_{\text {stab }}$.

Due to the applied pressure, all the contact elements are forced to be active along the interface. Employing a small value of the stabilization parameter (e.g. $\alpha_{\text {stab }}=0.01$ ) leads to a very strong enforcement of the geometric impenetrability condition, preventing the nodes to interpenetrate. Thus the interface cannot bent and the beams lock. On the other hand, a very large value of the stabilization parameter (e.g. $\left.\alpha_{\text {stab }}=10.0\right)$ displays only a very weak enforcement of the impenetrability condition. The beams do not lock, but the gaps between the two beams become intolerably large (see Fig. 17d). Applying a stabilization parameter in the range of $\alpha_{\text {stab }} \in$ [0.1-1.0], leads to a locking free behavior of the proposed contact strategy, while guaranteeing a sufficient enforcement of the geometric contact constraint. Fig. 18 displays the vertical displacement of node A (see Fig. 16) versus the applied moment. The results achieved with the sliding contact interface compare quite well with the conforming reference solution. Quantitative differences in the vertical displacements of point A seem to be a result of the rough triangulation of the beams with CST elements and not a direct consequence of the contact strategy. To demonstrate this, the example is analyzed again, using a finer discretization.

The initial as well as the deformed configuration for the conforming beam, using a fine mesh, is shown in Fig. 19a. Fig. 19b

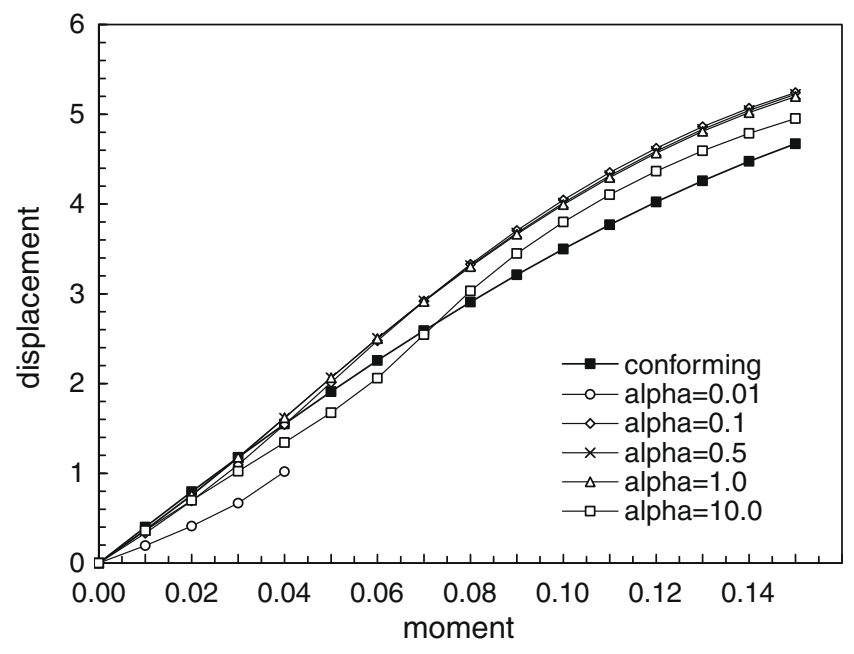

Fig. 18. Vertical displacement of Point A versus moment: rough mesh.

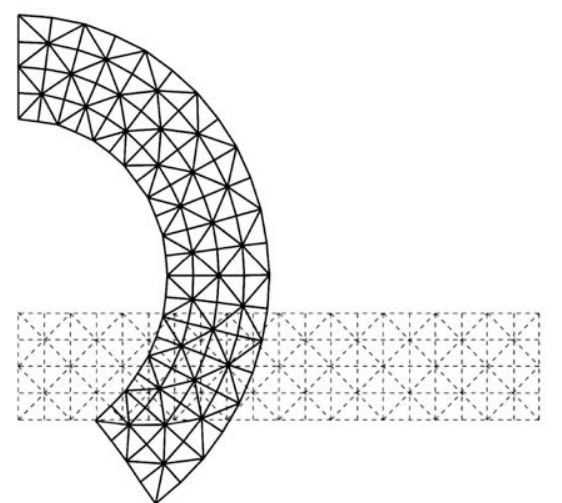

(a) conforming shows the results obtained with dissimilar meshes along the sliding interface, using a stabilization parameter of $\alpha_{\mathrm{stab}}=0.5$. The vertical displacement of node A versus the applied moment using a finer triangulation is shown in Fig. 20. All the results obtained with a sliding surface match very well with the conforming reference solution.

Thus the following conclusions can be drawn from this example:

- The proposed contact strategy displays a locking free behavior, utilizing a stability parameter in the range of $\alpha_{\text {stab }} \in[0.1-1.0]$.

- A too large value of $\alpha_{\text {stab }}$ results in a bad enforcement of the impenetrability constraint.

- A too small value of $\alpha_{\text {stab }}$ may lead to locking.

- The influence of $\alpha_{\text {stab }}$ on the results decreases with mesh refinement.

Due to these results, the following examples are analyzed using a stability parameter in the range of $\alpha_{\text {stab }} \in[0.1-1.0]$.

\subsection{Ironing}

In this example a frictional contact problem is analyzed, where both bodies undergo finite deformations. A block is pressed into an elastic slab and then slid over the surface. The slab is fixed at its

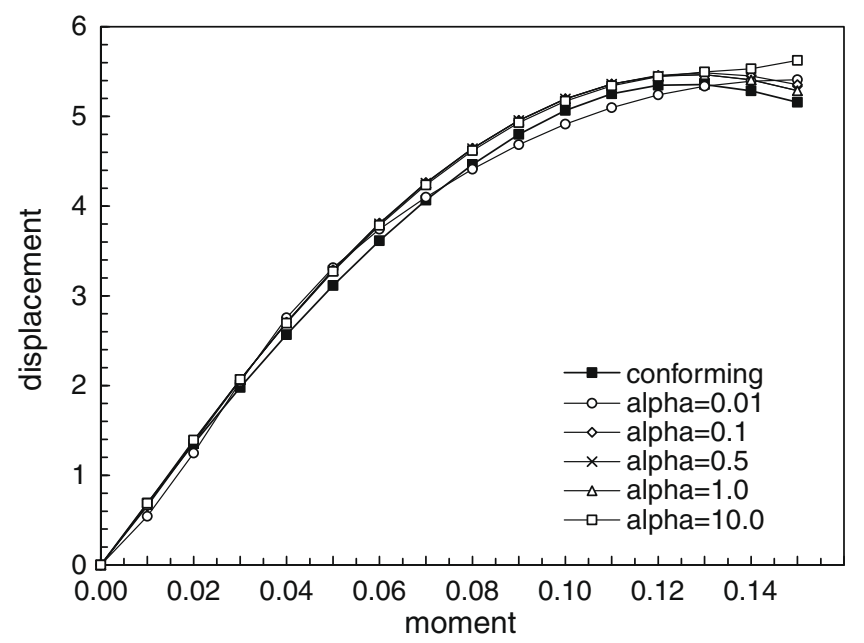

Fig. 20. Vertical displacement of Point A versus moment: fine mesh.

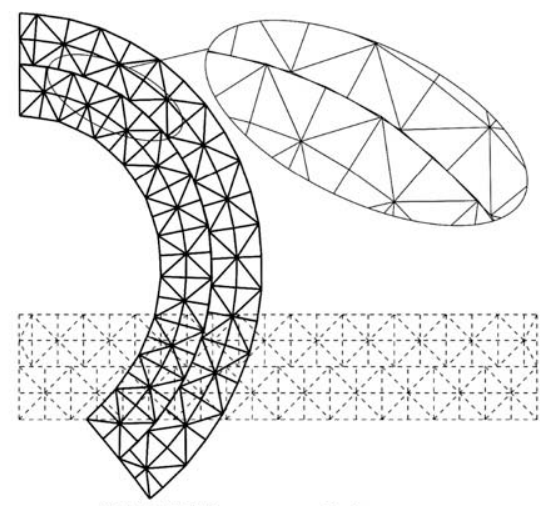

(b) CDM, $\alpha_{\text {stab }}=0.5$

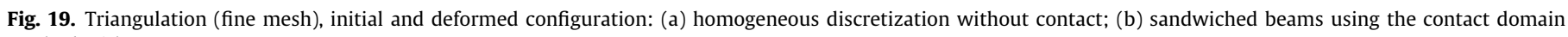
method with $\alpha_{\text {stab }}=0.5$. 
bottom and is 10 times softer than the indenting block. Vertical and horizontal displacements of the block are prescribed at its top edge. In the following two different ironing problems are discussed, which differ slightly in the geometry of the block and the amount of indentation $u_{V}$ of the punch into the slab (see Fig. 21). The material properties used for the block $\left(E_{b}=68.96 \times 10^{8}\left[\mathrm{~N} / \mathrm{mm}^{2}\right], v_{b}=0.32\right)$ and the slab $\left(E_{s}=68.96 \times 10^{7}\left[\mathrm{~N} / \mathrm{mm}^{2}\right], v_{s}=0.32\right)$ are the same in both cases. Friction is considered between all contacting faces, with the coefficient of friction being $\mu=0.3$. In both cases, the prescribed vertical displacement $u_{V}$ is applied between $t=0.0$ and 1.0 . The sub- sequent prescribed horizontal displacement is applied between $t=1.0$ and 2.0 , where the time has no physical meaning, as the calculation was carried out neglecting dynamical effects.

\subsubsection{Shallow ironing}

The first version of this example is analyzed in Fischer [6], Fischer and Wriggers [7] and Wriggers [17]. They use a mortar based contact formulation with a penalty regularization scheme together with quadratic 9-node-elements. As they report that the quality of the computed vertical and horizontal reactions depends
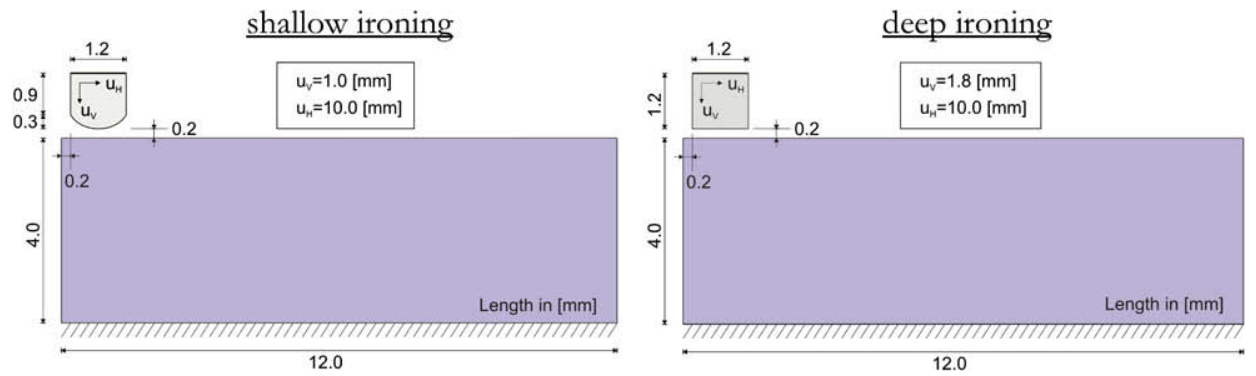

Fig. 21. Ironing: geometry for two different versions.

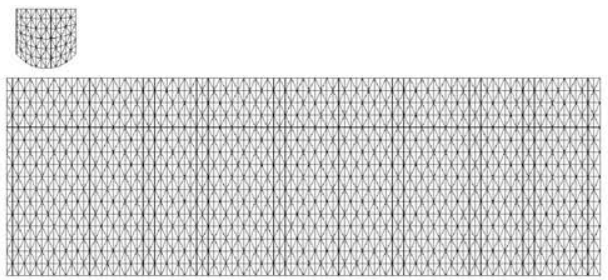

$\mathrm{t}=0.0$

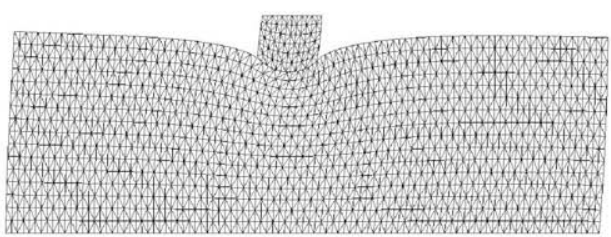

$\mathrm{t}=1.5$

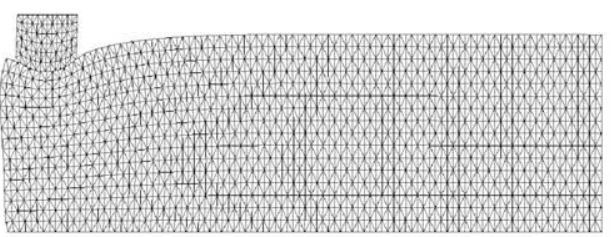

$\mathrm{t}=1.0$

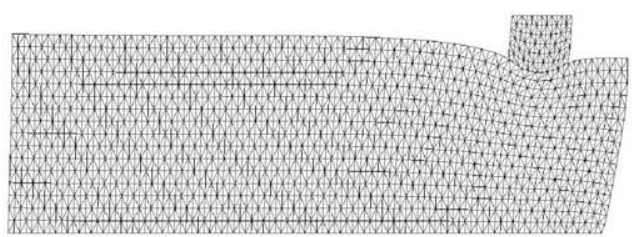

$\mathrm{t}=2.0$

Fig. 22. Shallow ironing: deformed configurations at different time steps.

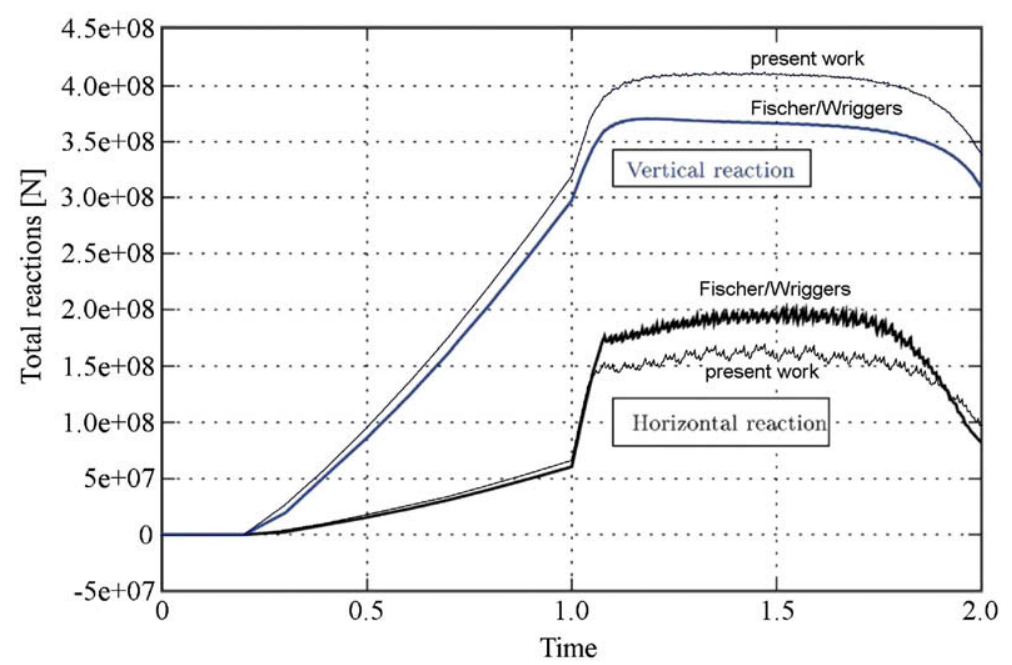

Fig. 23. Shallow ironing: computed total reaction forces versus time. 


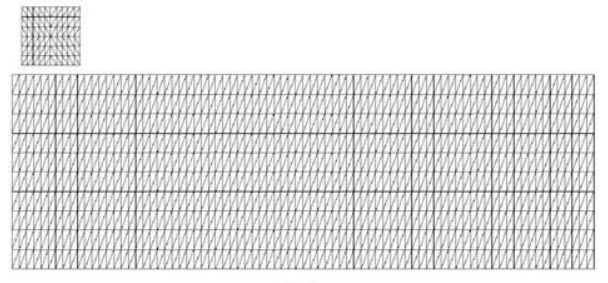

$\mathrm{t}=0.0$

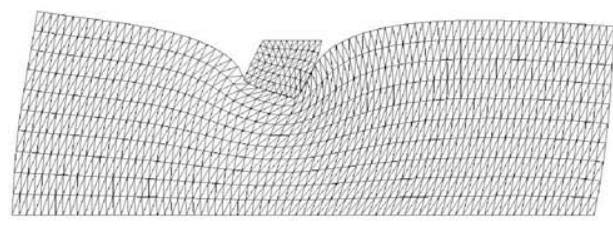

$\mathrm{t}=1.5$

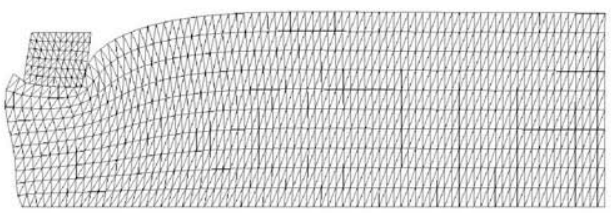

$\mathrm{t}=1.0$

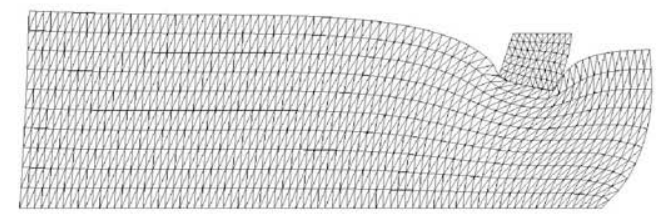

$\mathrm{t}=2.0$

Fig. 24. Deep ironing: deformed configurations at different time steps.

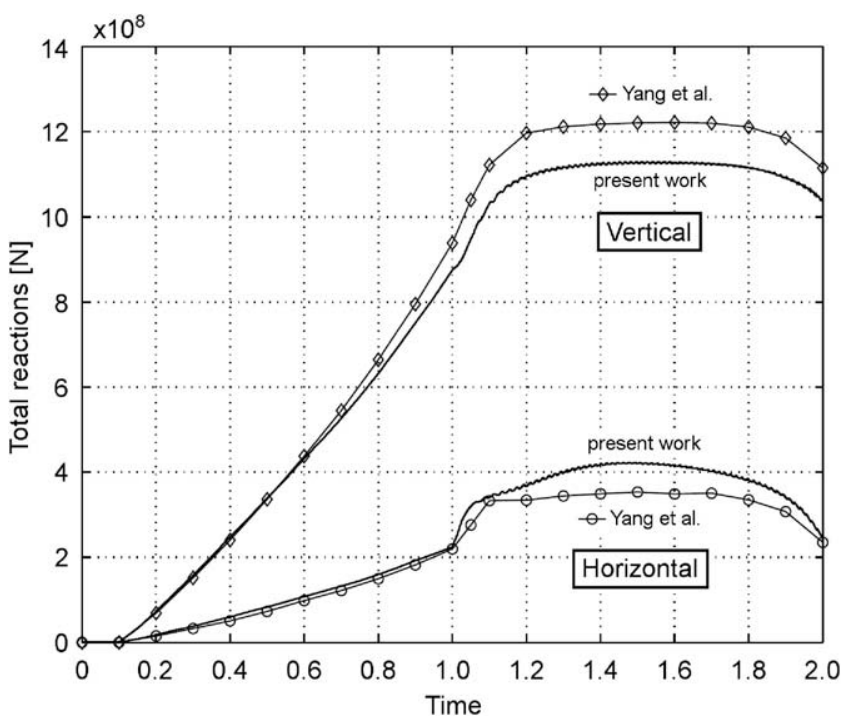

Fig. 25. Deep ironing: computed total reaction forces versus time. on the utilized finite element discretization, a comparable triangulation is used in the present study. Using the same number of finite element nodes, one quadratic 9-node-element is being replaced with eight linear 3-node-elements, which will lead to the same number of degrees of freedom involved in the problem. As in the reference works, the vertical displacement is applied in 10 time steps and the horizontal displacement is prescribed within 500 time steps. For the present calculation a stabilization parameter of $\alpha_{\text {stab }}=0.3$ is used.

The initial and three deformed configurations of the computation are shown in Fig. 22, which demonstrate the finite deformations involved in the ironing process. In Fig. 23 the total vertical and horizontal reaction forces, computed at the top of the indenting block, are plotted versus time. Together with the results of the present study, the graphs reported by Fischer and Wriggers [7] are displayed. While pressing the block into the slab, the curves are smooth and the two bodies stick together. Starting the horizontal movement, the vertical as well as the horizontal reaction forces increase until a limit is reached. At this stage the block starts sliding over the slab. While the vertical reaction force remains nearly constant, the horizontal reaction force oscillates. This is due to the fact

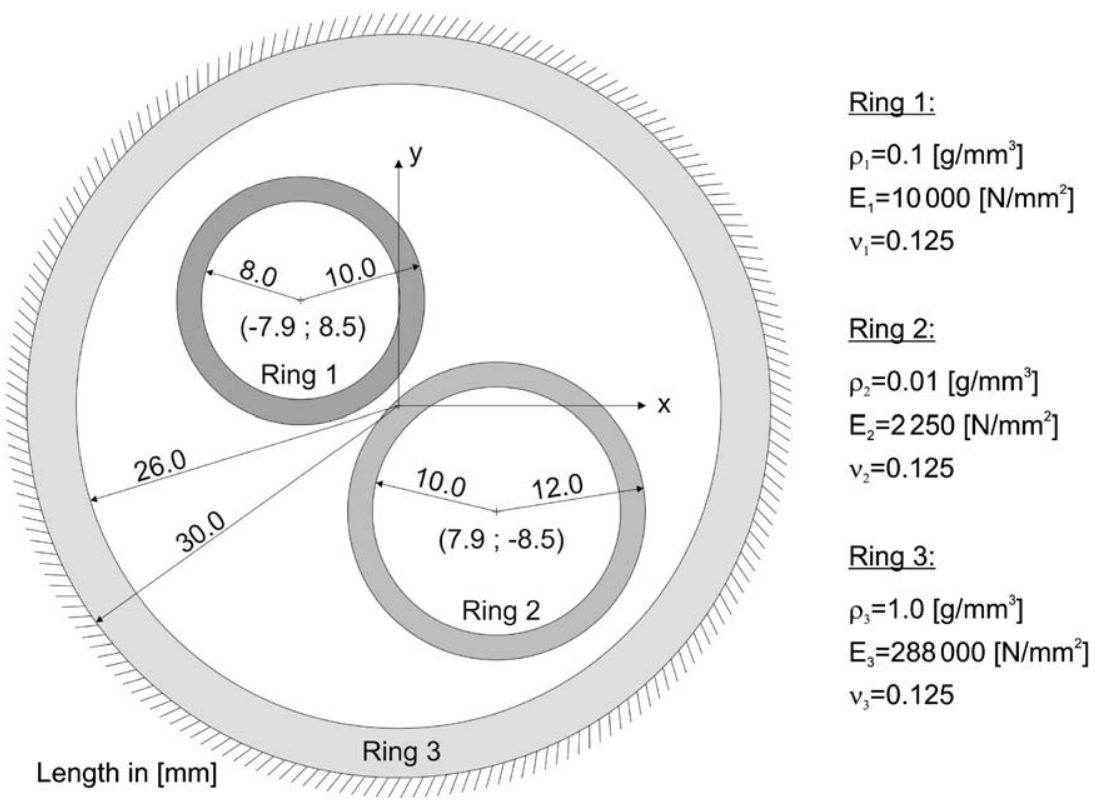

Fig. 26. Ring impact: geometry and material properties. 
that the finite element mesh of the slab has to slip around the right corner of the indenting block. Comparing the results of the present study with the ones reported in [7] one can note, that the qualitative behavior is very well captured, whereas the absolute values do not match exactly. Quantitative differences in the computed reaction forces may stem from the usage of different finite elements for the discretization of the contacting bodies. Due to Fischer and Wriggers [7] the computation of this problem using a node-to-segment approach fails around time step $t=1.2$. Thus, the present contact strategy behaves better than a classical node-to-segment formulation and produces confidential results compared with mortar based contact methods.

\subsubsection{Deep ironing}

The second version of the ironing problem is analyzed in Yang et al. [20], where they use again a large deformation mortar contact formulation. For two reasons, this version is a lot more demanding to compute than the first one. First, the geometry of the upper block is just a square, having sharp corners at the indenting side as well. This produces very large contact pressures at the lower right corner of the indenting block. Second, the amount of indentation is nearly twice as big as in the first version, making it very difficult for the finite elements in the slab to slide around the sharp edge of the punch. In fact, Yang et al. [20] report, that the analysis of this example with a node-to-segment formulation fails at $t=0.66$, being not even able to run the computation until the punch has fully indented.

In their calculation, Yang et al. [20] discretized the contacting bodies using bi-linear 4-node-elements. For reasons of comparison, again a comparable finite element triangulation is used for the present calculations, dividing one bi-linear 4-node-element into two linear 3-node-elements, leading to the same degrees of freedom. In [20] the number of load increments used for the vertical indentation and the horizontal sliding is not given. Here, the verti-
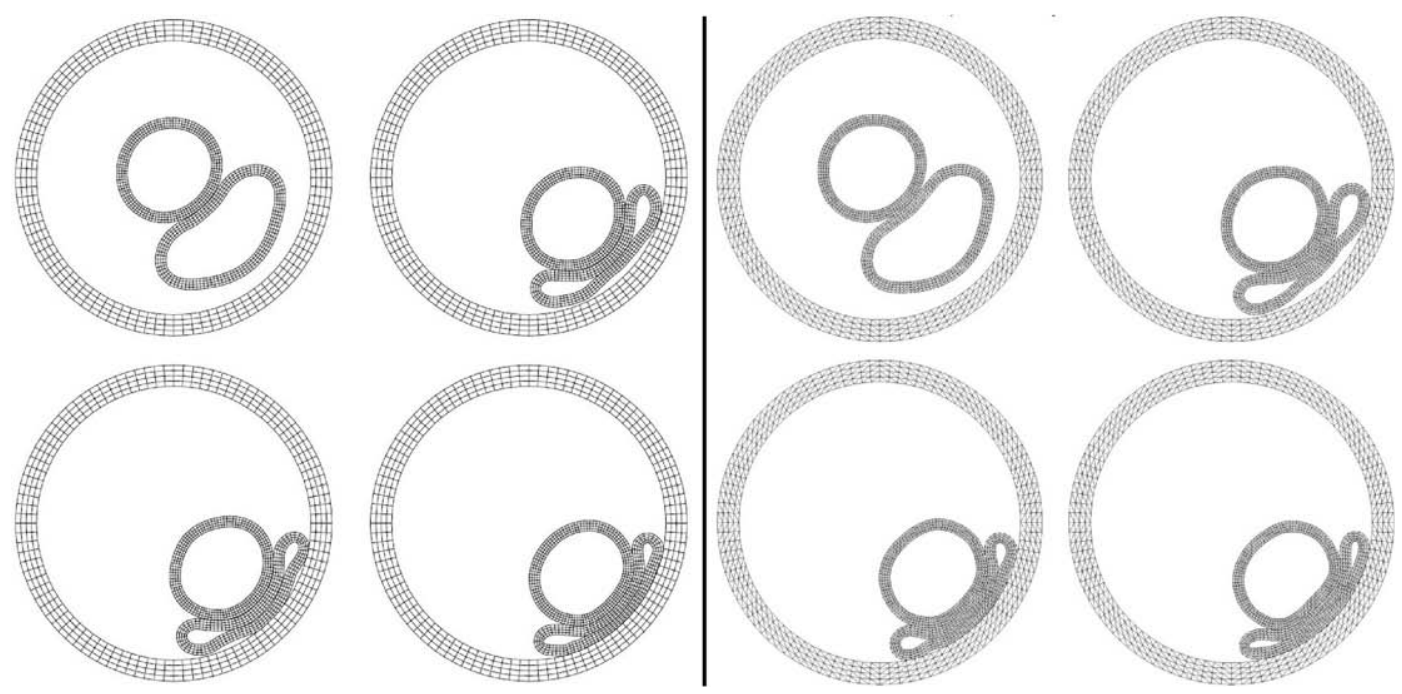

Fig. 27. Ring impact: comparison of deformed configurations at different time steps. Left: Yang and Laursen [19]/right: present work.

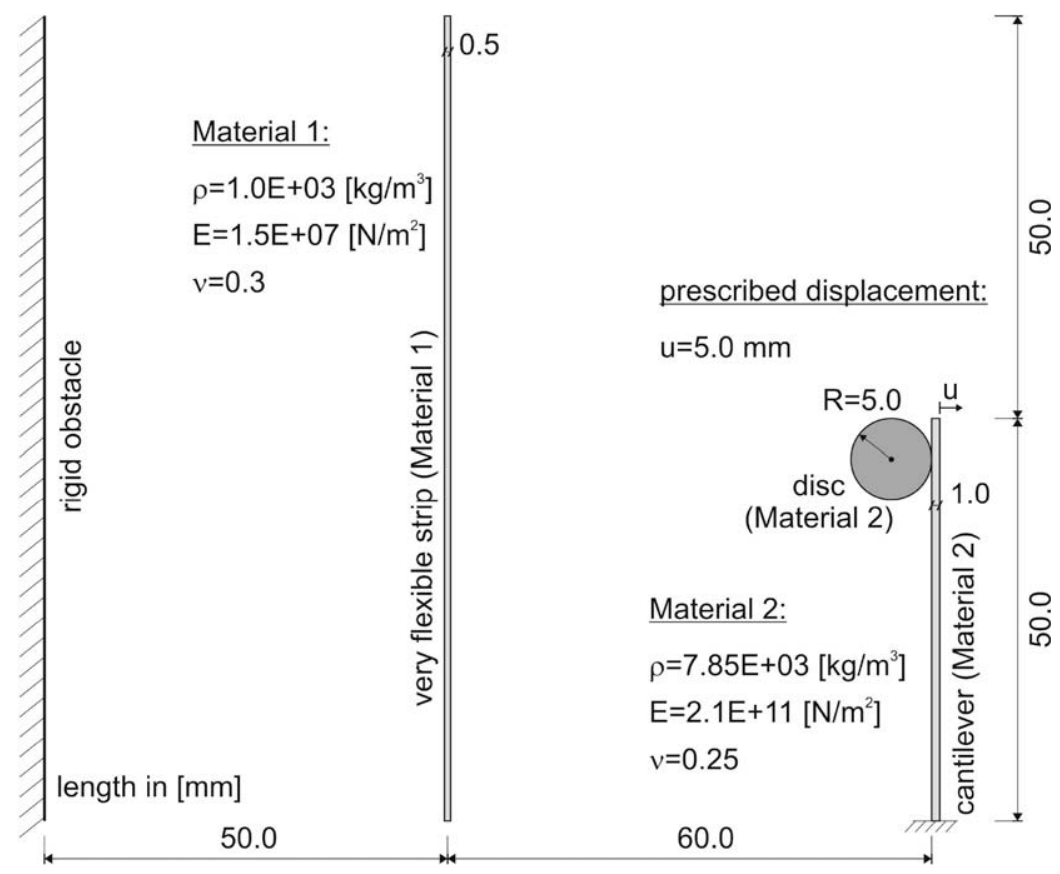

Fig. 28. Flipper: geometry and material properties. 
cal displacement is prescribed in 20 time steps and the horizontal displacement is applied in 1500 time steps. A stabilization parameter of $\alpha_{\text {stab }}=0.1$ is used.

The deformed configuration of the ironing problem is depicted in Fig. 24. In here, the appearance of finite deformations is even more pronounced than in the first version. The deformation of the slab is so large that even contact of the right side of the upper block has to be considered. In contrast to [20] frictional effects are considered in this part as well. Fig. 25 depicts again the total vertical and horizontal reactions, computed at the top edge of the punch. A similar behavior than in the first ironing version can be observed, but it seems that the amplitudes of the oscillating horizontal reactions are significant smaller. This is most probably due to the fact, that the horizontal displacements are applied in three times more displacement increments. The differences in the abso- lute values of the reaction forces are due to the usage of different finite elements for the discretization of the contacting bodies and due to the different treatment of the friction coefficient at the right side of the block with the slab.

\subsection{Ring impact}

This example was published by Yang and Laursen [19] to show the performance of their presented self-contact search algorithm. In this work, the example is chosen, to demonstrate, that the presented contact strategy can automatically deal with self-contact problems without any further modifications.

The geometry setup and the utilized material properties for the elastic rings are shown in Fig. 26. A fixed Dirichlet boundary condition is assigned to the external boundary of the largest ring (Ring

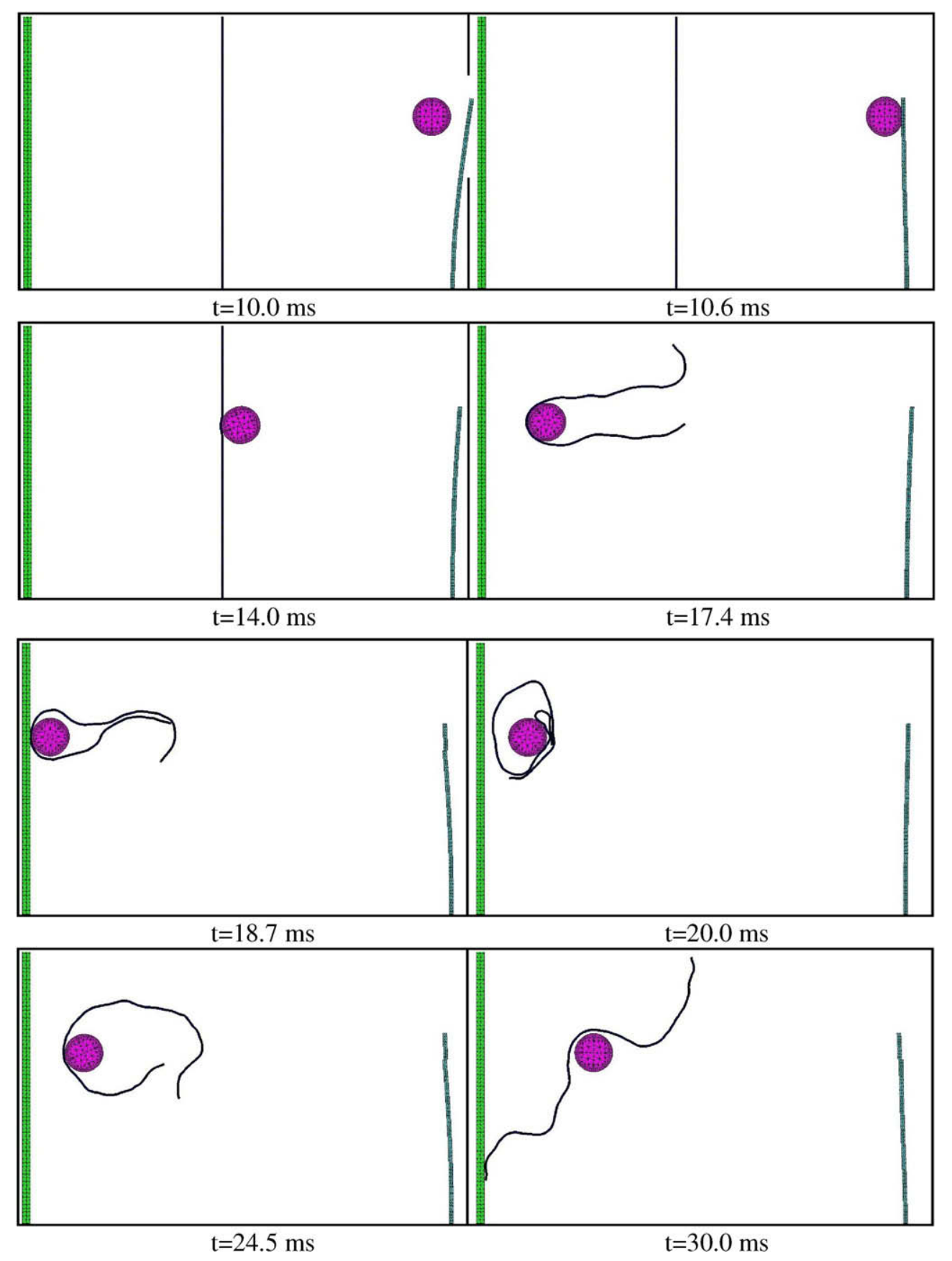

Fig. 29. Flipper - motion: without plotting the contact domain mesh. 
3). Two smaller rings are located within Ring 3, where the smallest ring (Ring 1) is given an initial velocity of $v=[30.0[\mathrm{~mm} /$ $\mathrm{ms}],-30.0[\mathrm{~mm} / \mathrm{ms}]]^{T}$ to hit the medium sized ring (Ring 2). After hitting Ring 2, the two inner rings move together towards the inner surface of Ring 3 . As the stiffness of the medium sized ring is very low, it gets pressed together between the two other rings in such a way, that self contact of the inner boundary of Ring 2 occurs. Between all possible contact pairings a frictional coefficient of $\mu=0.3$ is utilized. For the stabilization parameter a value of $\alpha_{\text {stab }}=0.1$ is chosen. To better compare the deformations with the ones shown in [19], the same time integration method and time step size are utilized. A Newmark time integration method is applied with $\beta=0.25$ and $\gamma=0.5$, which is included in the general framework of the Generalized- $\alpha$ method by setting the interpola- tion parameters to $\alpha_{f}=0.0$ and $\alpha_{m}=0.0$. The time step size is chosen to be constant with $\Delta t=0.001[\mathrm{~ms}]$.

In Fig. 27 a comparison of the deformed configurations at different time steps is shown. On the left hand side the results from Yang and Laursen [19] are plotted, who use a large deformation mortar contact method and on the right hand side the results from the present work are displayed. The results obtained with the newly developed contact domain method match very well with the ones obtained with the mortar method. In contrast to the "reference" solution, the present algorithm does not need a special contact search algorithm, optimized and adjusted for the treatment of self contact. The detection of possible contact pairs, including self contact, is automatically done with the contact pairing algorithm detailed in Section 3.1 without any modifications.
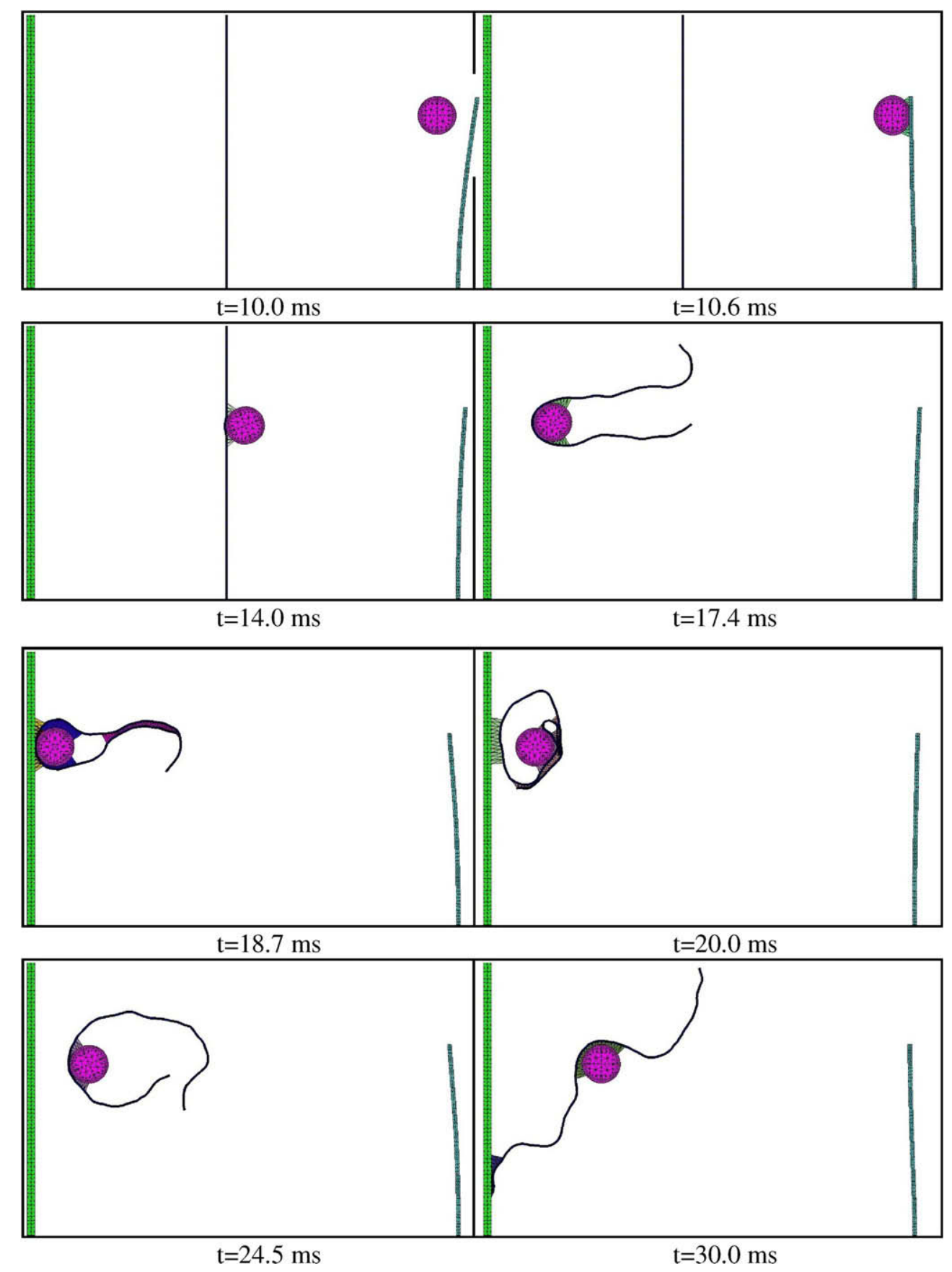

Fig. 30. Flipper - motion: with plotting the contact domain mesh. 


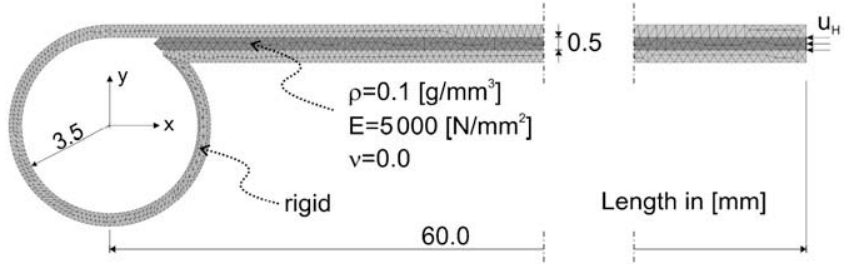

Fig. 31. Spiral: geometry and material properties.

\subsection{Flipper}

This example is devised to demonstrate the possibility to capture complex, highly dynamic contact scenarios including various regions with self-contact. In Fig. 28 the geometric setup as well as the material properties of the problem is shown. First the tip of the cantilever is moved horizontally by a prescribed displacement $u=5.0 \mathrm{~mm}$, which is then suddenly released at $t=10.0 \mathrm{~ms}$. Now the cantilever is free to move and starts swinging around its initial position. On the way back to its initial position, the cantilever hits the elastic disc, which will be accelerated towards the very flexible strip. When the disc gets into contact with the thin strip, this deforms significantly, such that it will get into contact with its own parts. Both the disc and the highly deformed strip are then hitting against the rigid wall, again producing a very difficult contact scenario. The dynamic analysis is performed using the Generalized- $\alpha$ scheme with a spectral radius of $\rho_{\infty}=0.85$, which introduces a little bit of numerical dissipation. As the numerical analysis is quite demanding, the time step size was adjusted several times during the calculation in order to capture the rapidly changing physics of the different contact scenarios (cantilever-todisc, disc-to-strip, strip-to-strip, strip-to-wall) and to handle the associated convergence difficulties. Friction is assumed between all possible contact pairs using $\mu=0.1$ and the stabilization parameter is set to $\alpha_{\text {stab }}=0.3$.
In Figs. 29 and 30 the complex motion of the problem is shown at different time steps, without and with displaying the constructed contact domain triangulation, respectively. The presented contact algorithm performs very well in this complex dynamic contact problem and can deal with self-contact without any additional difficulty by construction.

\subsection{Spiral}

In this problem, a thin strip is pushed through a tight form in order to be rolled up like a spiral. Therefore large relative tangential sliding as well as multiple self-contact has to be captured by the contact algorithm. The setup of the example, as well as the material properties of the strip, is shown in Fig. 31. Although the form is discretized with finite elements, it is assumed to be rigid and all its finite element nodes are fixed throughout the whole calculation. The strip is pushed by a prescribed horizontal displacement $u_{H}$ at its right edge. A dynamic analysis is carried out using the Generalized- $\alpha$ time integration scheme with a spectral radius of $\rho_{\infty}=1.0$. In a total calculation time of $t=60.0$ [ms] a horizontal displacement of $u_{H}=60.0[\mathrm{~mm}]$ is prescribed. A constant time step size is chosen to be $\Delta t=0.05[\mathrm{~ms}]$. In the analysis a stabilization parameter of $\alpha_{\text {stab }}=0.1$ is utilized. Between the contacting boundaries a frictionless contact behavior is assumed.

The deformed configurations of the spiral problem are shown in Fig. 32. It can be seen, that the contact strategy is able to capture the very large relative tangential sliding between the strip and the form as well as between different parts of the strip. Self contact of the strip, even being in contact on both sides of the strip at the same time, is tracked reliably by the present contact formulation.

\subsection{Multiple contact}

The last example is devised just to demonstrate, that the proposed contact strategy can handle contact scenarios between many
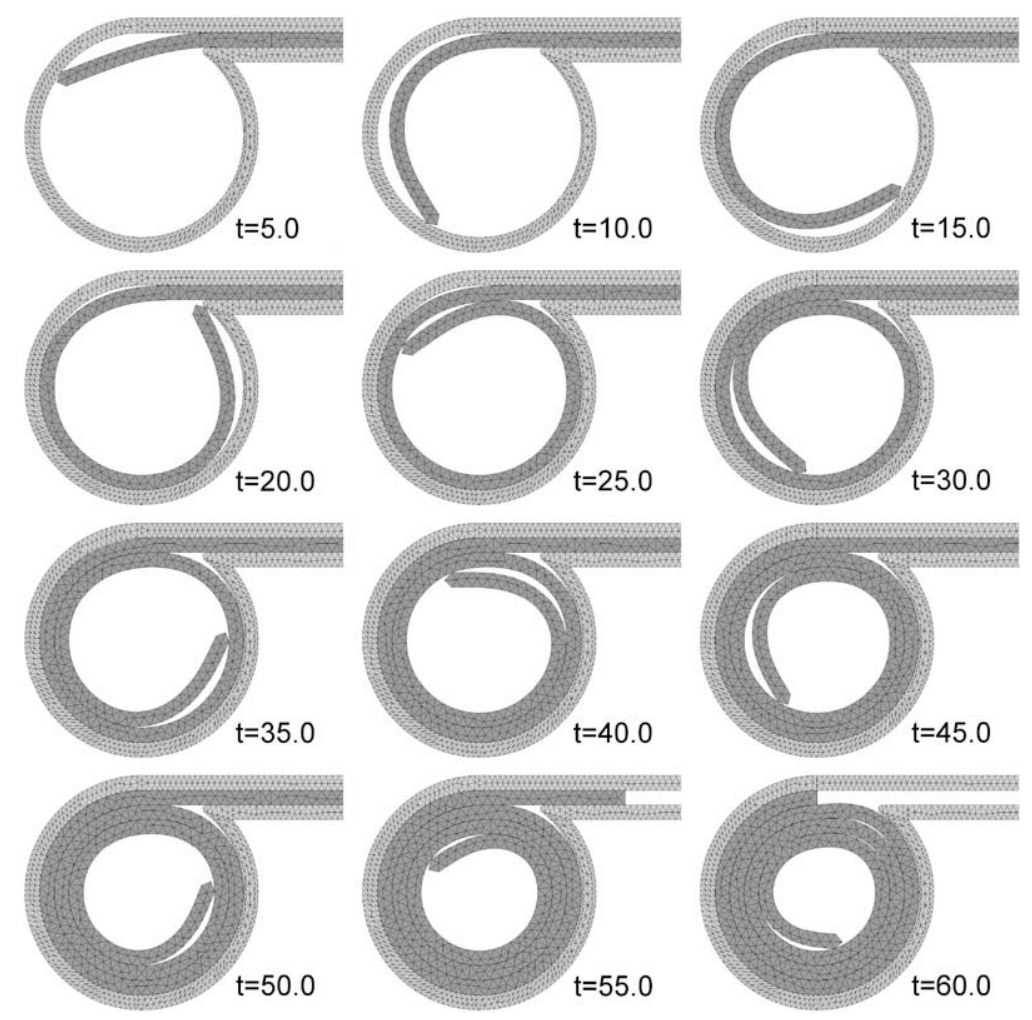

Fig. 32. Spiral: deformed configurations at different time steps ( $t$ in [ms]). 


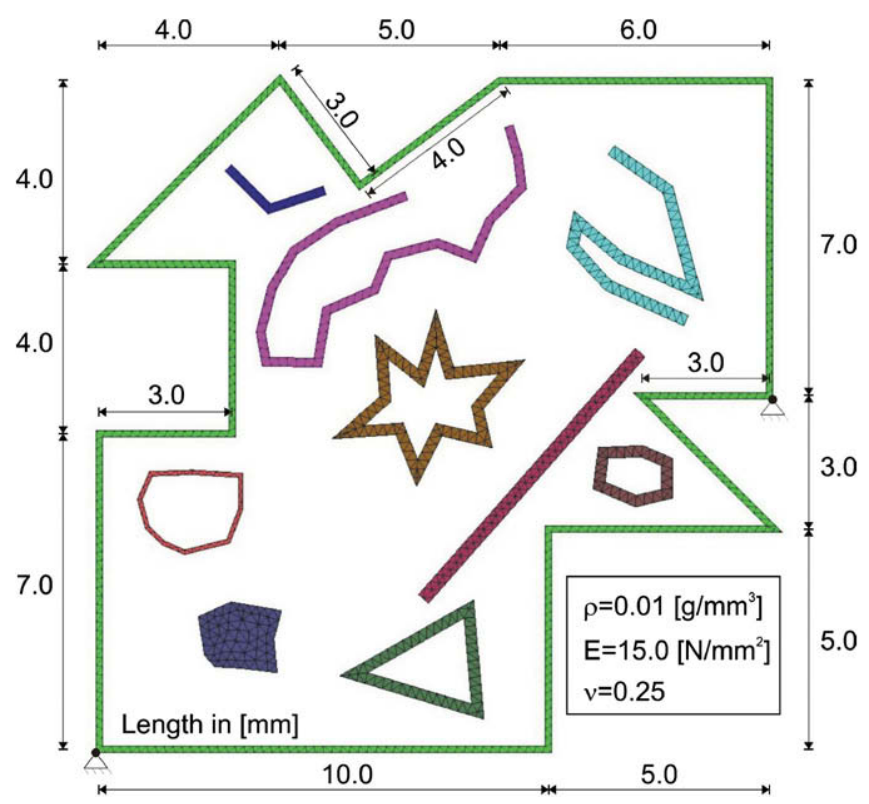

Fig. 33. Multiple contacts: geometry and material properties.

contacting bodies without any further modification. Nine geometrical objects are placed randomly as can be seen in Fig. 33 inside an elastic boundary. All objects and the boundary have the same elastic properties, given in Fig. 33. Some of the objects are arbitrarily accelerated to get into contact with the others. The dynamic calculation is done using the Generalized- $\alpha$ scheme with $\rho_{\infty}=1.0$, a stabilization parameter $\alpha_{\text {stab }}=0.3$, a friction coefficient of $\mu=0.2$ between all occurring contact pairs and a constant time step of $\Delta t=0.01[\mathrm{~ms}]$.

In Fig. 34 the deformed configurations of the multiple contact problem at different time steps are depicted. The contact algorithm can handle at no difficulty the contact of sharp edges as well as multiple contact pairings of one object with others and with itself. This underlines the powerful contact pairing strategy introduced in Section 3.1.

\section{Concluding remarks}

Together with the first part of this paper [14] a new contact strategy is developed to analyze two-dimensional, large deforma- tion, static and dynamic (self) contact problems. Compared to many existing contact formulations, the presented contact domain method differs in many significant ways. The present formulation produces a solution, which does not dependent on the choice of slave and master sides, as the contact pairing is uniquely defined via a constraint Delaunay triangulation. Thus, no projections of slave nodes/segments onto master segments have to be performed, circumventing the problem of possible pathological cases. As the Lagrange multipliers are introduced on the newly defined contact domain, the sometimes quite cumbersome numerical procedure to evaluate integrals of products of shape functions living on different surface grids involved in the mortar method is omitted. Due to the element-wise constant approximation of the Lagrange multipliers, the stabilized constraint equations can be decoupled and the necessary integrations can be performed analytically. This allows to condense the discrete Lagrange multipliers locally on element level which eases the implementation of the contact algorithm and speeds up the computation. The utilized contact pairing strategy displays a unified approach in the context of contact searching algorithms, which does not necessitate any modifications in the treatment of self contact or multiple contacts.

The introduced stabilization terms in the variational constraint equations lead to non-symmetric contact stiffness matrices even for the normal contact and stick part, which could be symmetrized, utilizing additional stabilization terms in the mechanical virtual work expression [8]. Typical for Nitsche type methods, the computation of the contact contributions depend on the stresses in the contacting bodies. Consequently, the resulting contact stiffnesses contribute as well to degrees of freedom of the finite elements at the contact boundary, which slightly increases the bandwidth of the final matrices. Furthermore, the linearization of the stresses at the boundaries of the contacting bodies depends upon the constitutive law and the type of finite element used therein. That means, that the implementation of the proposed contact strategy cannot be as general as one based on classical Lagrange multiplier or penalty methods.

Numerous numerical examples presented in Section 6 have shown that the contact domain method will produce reliable and accurate results. The proposed contact strategy exactly passes the contact patch test, independent of the value of the utilized stabilization parameter (see Section 6.1), which is a direct consequence of the usage of a contact domain, approximated with a full set of non-overlapping contact patches. Section 6.3 demonstrates the
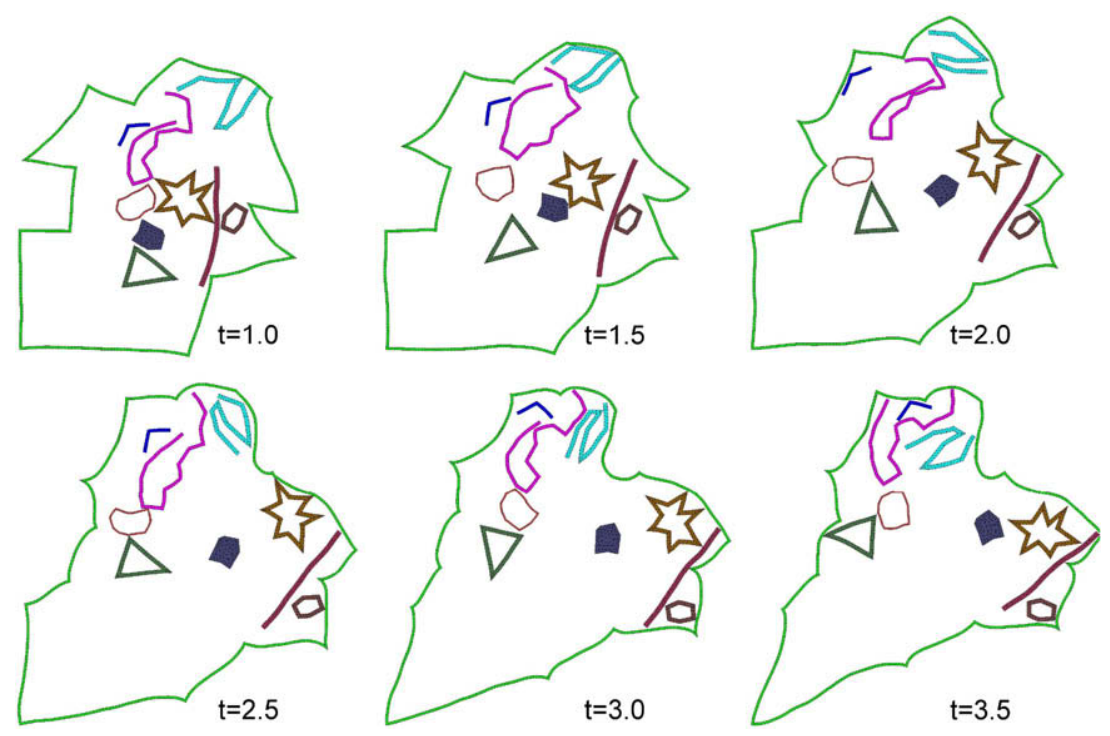

Fig. 34. Multiple contact: deformed configurations at different time steps ( $t$ in [ms]). 
locking free behavior of the proposed method, when using appropriate values of the stabilization parameter $\alpha_{\text {stab. }}$. From this, and from the experience in numerous numerical examples, a stabilization parameter $\alpha_{\text {stab }} \in[0.1-1.0]$ can be recommended to produce very good results for most applications. Challenging problems, so far only captured by recently developed mortar based methods as well as highly dynamic problems involving multiple contacts, including self contact, can be analyzed without additional modification of the algorithm. The consistent linearization of the contact contributions, detailed in Section 4.6, guarantees a quadratic convergence within the Newton iteration, once the active set has converged. This can be confirmed for the numerical calculations in Section 6.

Despite the necessary linearization of the first Piola-Kirchhoff stress tensor, the implementation of this contact method is quite simple. The introduced stabilization terms, together with an element-wise constant approximation of the introduced Lagrange multipliers allow the local elimination of the discrete Lagrange multipliers on element level. All the necessary integrations can be done analytically, circumventing possible difficulties in performing demanding numerical quadratures. Furthermore, the calculation of the contact forces and the contact stiffness contributions can be carried out locally for every individual contact domain element, which only necessitates some information of the adjacent "body" element. Therefore a parallel implementation of this contact algorithm seems to be quite natural and simple to undertake which could even speed up this contact algorithm.

As the performance of the presented contact strategy seems to be superior to classical node-to-segment formulations and comparable to recently developed mortar based contact algorithms, the proposed contact domain method displays a sound alternative in the field of computational contact mechanics. However, further investigations, especially for three-dimensional applications are necessary, which will be the scope of future work.

\section{Acknowledgements}

The first author gratefully acknowledges the support of the German Research Foundation through the grant HA 5433/1-1. Furthermore, the financial support from the Spanish Ministry of Science and Technology, through grant BIA2005-09250-C03-03, and the Spanish Ministry of Science and Innovation, through grant BIA2008-00411 are gratefully acknowledged.

\section{Appendix A. Linearization of first Piola-Kirchhoff stresses}

The first Piola-Kirchhoff stress tensor $\mathbf{P}$ can be written in terms of the material deformation gradient $\mathbf{F}$ and the second Piola-Kirchhoff stress tensor $\mathbf{S}[2]$

$\mathbf{P}=\mathbf{F} \cdot \mathbf{S}$.

Its linearization yields

$\Delta \mathbf{P}=\Delta \mathbf{F} \cdot \mathbf{S}+\mathbf{F} \cdot \Delta \mathbf{S}$

with the linearization of the second Piola-Kirchhoff stress tensor

$\Delta \mathbf{S}=\mathbb{C}^{\text {tang }}: \Delta \mathbf{E}$,

where $\mathbb{C}^{\text {tang }}$ is the fourth order constitutive tangent operator, which relates the incremental second Piola-Kirchhoff stresses $\Delta \mathbf{S}$ with the incremental non-linear Green-Lagrange strains $\Delta \mathbf{E}$.

\section{A.1. Matrix notation}

Using a two-dimensional, 3-noded finite element formulation for the discretization of the "body" elements, the linearization of the first Piola-Kirchhoff stress tensor can be written in the matrix formulation. With the definition of the vector of incremental nodal displacements in Eq. (74), based on the node numbering given in Fig. 7, the following vectors and matrices are defined:

The linearization of the material deformation gradient

$\Delta \overline{\mathbf{F}}_{(4 \times 1)}=\left(\begin{array}{c}\Delta F_{11} \\ \Delta F_{22} \\ \Delta F_{12} \\ \Delta F_{21}\end{array}\right)=\widetilde{\mathbf{B}}_{(4 \times 8)} \Delta \mathbf{d}_{(8 \times 1)}$

with the linear B-operator matrix

$\widetilde{\mathbf{B}}_{(4 \times 8)}=\left(\begin{array}{cccccccc}N_{1,1} & 0 & N_{2,1} & 0 & 0 & 0 & N_{4,1} & 0 \\ 0 & N_{1,2} & 0 & N_{2,2} & 0 & 0 & 0 & N_{4,2} \\ N_{1,2} & 0 & N_{2,2} & 0 & 0 & 0 & N_{4,2} & 0 \\ 0 & N_{1,1} & 0 & N_{2,1} & 0 & 0 & 0 & N_{4,1}\end{array}\right)$,

where $N_{I, J}$ are the derivatives of the shape functions $N_{I}$ in the adjacent "body" element, with respect to the coordinate at the previous configuration.

The linearization of the second Piola-Kirchhoff stress tensor

$$
\begin{aligned}
\Delta \overline{\mathbf{S}}_{(4 \times 1)}= & \left(\begin{array}{c}
\Delta S_{11} \\
\Delta S_{22} \\
\Delta S_{12} \\
\Delta S_{21}
\end{array}\right)=\mathbf{D}_{(4 \times 4)} \Delta \overline{\mathbf{E}}_{(4 \times 1)}=\mathbf{D}_{(4 \times 4)}\left(\begin{array}{c}
\Delta E_{11} \\
\Delta E_{22} \\
\Delta E_{12} \\
\Delta E_{21}
\end{array}\right) \\
& =\mathbf{D}_{(4 \times 4)} \mathbf{B}_{(4 \times 8)} \Delta \mathbf{d}_{(8 \times 1)}
\end{aligned}
$$

with the incremental constitutive tangent matrix

$$
\mathbf{D}_{(4 \times 4)}=\left(\begin{array}{llll}
\mathbb{C}_{1111}^{\text {tang }} & \mathbb{C}_{1122}^{\text {tang }} & \mathbb{C}_{1112}^{\text {tang }} & \mathbb{C}_{1121}^{\text {tang }} \\
\mathbb{C}_{2211}^{\text {tang }} & \mathbb{C}_{2222}^{\text {tang }} & \mathbb{C}_{2212}^{\text {tang }} & \mathbb{C}_{2221}^{\text {tang }} \\
\mathbb{C}_{1211}^{\text {tang }} & \mathbb{C}_{1222}^{\text {tang }} & \mathbb{C}_{1212}^{\text {tang }} & \mathbb{C}_{1221}^{\text {tang }} \\
\mathbb{C}_{2111}^{\text {tang }} & \mathbb{C}_{2122}^{\text {tang }} & \mathbb{C}_{2112}^{\text {tang }} & \mathbb{C}_{2121}^{\text {tang }}
\end{array}\right)
$$

and the non-linear B-operator matrix

$$
\mathbf{B}_{(4 \times 8)}=\left(\begin{array}{llll}
\mathbf{B}_{1} & \mathbf{B}_{2} & \mathbf{0}_{(4 \times 2)} & \mathbf{B}_{4}
\end{array}\right)
$$

with

$$
\mathbf{B}_{I}=\left(\begin{array}{cc}
F_{11} N_{I, 1} & F_{21} N_{I, 1} \\
F_{12} N_{I, 2} & F_{22} N_{I, 2} \\
\frac{1}{2}\left(F_{11} N_{I, 2}+F_{12} N_{I, 1}\right) & \frac{1}{2}\left(F_{21} N_{I, 2}+F_{22} N_{I, 1}\right) \\
\frac{1}{2}\left(F_{11} N_{I, 2}+F_{12} N_{I, 1}\right) & \frac{1}{2}\left(F_{21} N_{I, 2}+F_{22} N_{I, 1}\right) .
\end{array}\right) .
$$

Furthermore, the values of the material deformation gradient and the second Piola-Kirchhoff stress tensor are organized in matrices:

$$
\begin{aligned}
\overline{\mathbf{F}}_{(4 \times 4)} & =\left(\begin{array}{cccc}
F_{11} & 0 & 0 & F_{12} \\
0 & F_{22} & F_{21} & 0 \\
0 & F_{12} & F_{11} & 0 \\
F_{21} & 0 & 0 & F_{22}
\end{array}\right) \text { and } \\
\overline{\mathbf{S}}_{(4 \times 4)} & =\left(\begin{array}{cccc}
S_{11} & 0 & S_{21} & 0 \\
0 & S_{22} & 0 & S_{12} \\
S_{12} & 0 & S_{22} & 0 \\
0 & S_{21} & 0 & S_{11}
\end{array}\right)
\end{aligned}
$$

Thus the linearization of the first Piola-Kirchhoff stress tensor is given in matrix form:

$$
\Delta \overline{\mathbf{P}}_{(4 \times 1)}=\left(\begin{array}{c}
\Delta P_{11} \\
\Delta P_{22} \\
\Delta P_{12} \\
\Delta P_{21}
\end{array}\right)=\left(\overline{\mathbf{S}}_{(4 \times 4)} \widetilde{\mathbf{B}}_{(4 \times 8)}+\overline{\mathbf{F}}_{(4 \times 4)} \mathbf{D}_{(4 \times 4)} \mathbf{B}_{(4 \times 8)}\right) \Delta \mathbf{d}_{(8 \times 1)} .
$$




\section{References}

[1] D. Benson, J.A. Hallquist, Single surface contact algorithm for the post-buckling analysis of shell structures, Comput. Method Appl. Mech. Engrg. 78 (1990) 141-163.

[2] J. Bonet, R.D. Wood, Nonlinear Continuum Mechanics for Finite Element Analysis, Cambridge University Press, Cambridge, UK, 1997.

[3] J. Chung, G.M.A. Hulbert, Time integration algorithm for structural dynamics with improved numerical dissipation: the Generalized-alpha method, J. Appl. Mech. 60 (1993) 371-375.

[4] M.A. Crisfield, Re-visiting the contact patch test, Int. J. Numer. Method Engrg. 48 (2000) 435-449.

[5] N. El-Abbasi, K.-J. Bathe, Stability and patch test performance of contact discretizations and a new solution algorithm, Comput. Struct. 79 (2001) 1473 1486.

[6] K.A. Fischer, Mortar Type Methods Applied to Nonlinear Contact Mechanics, Ph.D. Thesis, Research Report F05/2, Institut für Baumechanik und Numerische Mechanik, Universtität Hannover, Germany, 2005.

[7] K.A. Fischer, P. Wriggers, Mortar based frictional contact formulation for higher order interpolations using the moving friction cone, Comput. Method Appl Mech. Engrg. 195 (2006) 5020-5036.

[8] P. Heintz, P. Hansbo, Stabilized Lagrange multiplier method for bilateral elastic contact with friction, Comput. Method Appl. Mech. Engrg. 195 (2006) 4323 4333.

[9] H. Hertz, Über die Berührung fester elastischer Körper, Journal für die reine und angewandte Mathematik 92 (1881) 156-171.
[10] S.R. Idelsohn, E. Onate, N. Calvo, F. Del Pin, The meshless finite element method, Int. J. Numer. Method Engrg. 58 (2003) 893-912.

[11] T.A. Laursen, Computational Contact and Impact Mechanics, Springer, 2002.

[12] N. Newmark, A method of computation for structural dynamics, J. Engrg. Mech. Div. 85 (1959) 67-94.

[13] M. Oldenburg, N. Larsgunnar, The position code algorithm for contact searching, Int. J. Numer. Method Engrg. 37 (1994) 359-386.

[14] J. Oliver, S. Hartmann, J.C. Cante, R. Weyler, J. Hernández, A contact domain method for large deformation frictional contact problems. Part 1: Theoretical basis, Comput. Method Appl. Mech. Engrg. 198 (2009) 2591-2606.

[15] M. Puso, T. Laursen, A mortar segment-to-segment contact method for large deformation solid mechanics, Comput. Method Appl. Mech. Engrg. 193 (2004) 601-629.

[16] M. Puso, T. Laursen, A mortar segment-to-segment frictional contact method for large deformations, Comput. Method Appl. Mech. Engrg. 193 (2004) 48914913.

[17] P. Wriggers, Computational Contact Mechanics, second ed., Springer, Berlin/ Heidelberg/New York, 2006.

[18] B. Yang, T. Laursen, A contact searching algorithm including bounding volume trees applied to finite sliding mortar formulations, Comput. Mech. 41 (2008) 189-205.

[19] B. Yang, T. Laursen, A large deformation mortar formulation of self contact with finite sliding, Comput. Method Appl. Mech. Engrg. 197 (2008) 756-772.

[20] B. Yang, T. Laursen, X. Meng, Two-dimensional mortar contact methods for large deformation frictional sliding, Int. J. Numer. Method Engrg. 62 (2005) 1183-1225. 